\title{
Entanglement and particle correlations of Fermi gases in harmonic traps
}

\author{
Ettore Vicari \\ Dipartimento di Fisica dell'Università di Pisa and INFN, Pisa, Italy
}

(Dated: October 17, 2018)

\begin{abstract}
We investigate quantum correlations in the ground state of noninteracting Fermi gases of $N$ particles trapped by an external space-dependent harmonic potential, in any dimension. For this purpose, we compute one-particle correlations, particle fluctuations and bipartite entanglement entropies of extended space regions, and study their large- $N$ scaling behaviors. The half-space von Neumann entanglement entropy is computed for any dimension, obtaining $S_{\mathrm{HS}} \approx c_{l} N^{(d-1) / d} \ln N$, analogously to homogenous systems, with $c_{l}=1 / 6,1 /(6 \sqrt{2}), 1 /(6 \sqrt{6})$ in one, two and three dimensions respectively. We show that the asymptotic large- $N$ relation $S_{A} \approx \pi^{2} V_{A} / 3$, between the von Neumann entanglement entropy $S_{A}$ and particle variance $V_{A}$ of an extended space region $A$, holds for any subsystem $A$ and in any dimension, analogously to homogeneous noninteracting Fermi gases.

PACS numbers: 03.65.Ud, 05.30.Fk, 03.67.Mn
\end{abstract}

\section{INTRODUCTION}

The recent developments in the experiments of dilute atom gases, namely the achievement of Bose-Einstein condensation in dilute atomic vapors of ${ }^{87} \mathrm{Rb}$ and ${ }^{23} \mathrm{Na}[1$, 2] and the great progress in the experimental manipulation of cold atoms in optical lattices [3] have provided a great opportunity to investigate the interplay between quantum and statistical behaviors in many-body systems. A common feature of these experiments is the presence of a confining harmonic potential which traps the particles within a limited spatial region. The capability of varying the confining potential, which may also depend on the spatial directions, allows also to vary the effective spatial geometry of the particle systems, including quasi-1D geometries, see, e.g., Refs. [4 9].

In this paper we investigate the quantum correlations arising within the ground state of noninteracting Fermi gases trapped by an external space-dependent harmonic potential. Quantum correlations can be characterized by the expectation values of the products of local operators, such as the particle density and one-particle operators, or by their integral over a space region $A$, such as the particle-number fluctuations within $A$. Quantum correlations are also characterized by the fundamental phenomenon of entanglement, which gives rise to nontrivial connections between different parts of extended quantum systems 10 12]. A measure of entanglement is achieved by computing von Neumann (vN) or Rényi entanglement entropies of the reduced density matrix of a subsystem. One-particle correlations and bipartite entanglement entropies provide important and complemetary information of the quantum behavior of many-body systems, because they probe different features of the quantum dynamics.

We consider Fermi gases of $N$ particles confined by harmonic traps of arbitrary dimension, and study the large- $N$ scaling behavior of the above-mentioned observables to characterize the quantum correlations of the ground state. We determine the asymptotic behaviors of the half-space entanglement entropies in any dimension, which turn out to increase as $N^{(d-1) / d} \ln N$, analogously to homogenous systems [13]. We study the relation between particle fluctuations and entanglement entropies of extended space regions. This is motivated by recent proposals of considering the particle fluctuations as effective probes of many-body entanglement at zero temperature [14 19], which are more easily accessible experimentally. In homogeneous finite-volume systems of noninteracting fermions, of any dimension, the vN entanglement entropy $S_{A}$ of an extended subsystem $A$ turns out to be closely related to the particle variance $V_{A}$ within $A$. Indeed, asymptotically for a large number of particles $N, S_{A} / V_{A} \approx \pi^{2} / 3$ for any subsystem $A$ and in any dimension [19], with $O(1 / \ln N)$ corrections. We show that this asymptotic behavior also holds in the presence of a space-dependent harmonic potential, such as the one which characterizes recent experiments with cold atoms. For this purpose we present several analytical results in the large- $N$ limit, and numerical (practically exact) results at fixed $N$ by computations from the ground-state wave function.

The paper is organized as follows. Sec. II reports some general expressions for the ground-state many-body wave function of free fermion gases in a harmonic trap, and define the observables that we consider. Sec. III focuses on one-dimensional (1D) systems. Systems in higher dimensions are considered in Sec. IV. Finally, in Sec. V] we summarize our main results and draw our conclusions.

\section{GROUND STATE AND OBSERVABLES OF TRAPPED FREE FERMION GASES}

\section{A. The ground state in a harmonic trap}

We consider a gas of $N$ noninteracting spinless fermionic particles of mass $m$ confined within a limited space region by an external potential. In the following we set $\hbar=1$ and $m=1$. The ground-state wave function is

$$
\Psi\left(\mathbf{x}_{1}, \ldots, \mathbf{x}_{N}\right)=\frac{1}{\sqrt{N !}} \operatorname{det}\left[\psi_{i}\left(\mathbf{x}_{j}\right)\right]
$$


where $\psi_{i}$ are the lowest $N$ eigensolutions of the oneparticle Schrödinger equation

$$
H \psi_{i}=E_{i} \psi_{i}, \quad H=\frac{\mathbf{p}^{2}}{2}+V(\mathbf{x}) .
$$

A generic power-law rotational-invariant potential such as

$$
V(\mathbf{x})=\frac{1}{p}\left(\frac{\mathbf{x}^{2}}{l^{2}}\right)^{p / 2}
$$

where $l$ is the trap size, gives rise to a trap length scale $\xi$ which behaves as a nontrivial power of $l$,

$$
\xi \equiv l^{\theta}, \quad \theta=\frac{p}{p+2},
$$

where $\theta$ is the trap exponent [20], which does not depend on the spatial dimension in free fermion gases. The power $p=2$ describes the harmonic trap, where $\xi$ is the socalled oscillator length. In the limit $p \rightarrow \infty$ the system becomes equivalent to a Fermi gas confined by a hardwall spherical trap of radius $l$.

The one-particle energy spectrum in harmonic traps is discrete. The eigensolutions can be written as a product of eigenfunctions of corresponding 1D Schr̈odinger problems, i.e.

$$
\begin{aligned}
& \psi_{n_{1}, n_{2}, \ldots, n_{d}}(\mathbf{x})=\prod_{i=1}^{d} \phi_{n_{i}}\left(x_{i}\right), \\
& E_{n_{1}, n_{2}, \ldots, n_{d}}=\sum_{i=1}^{d} e_{n_{i}},
\end{aligned}
$$

where the $n_{i}$ label the eigenfunctions along the $d$ directions, which are

$$
\begin{aligned}
& \phi_{n}(x)=\xi^{-1 / 2} \frac{H_{n-1}(X)}{\pi^{1 / 4} 2^{(n-1) / 2}(n-1) ! 1 / 2} e^{-X^{2} / 2}, \\
& e_{n}=\xi^{-2}(n-1 / 2), \quad n=1,2, \ldots
\end{aligned}
$$

where $X=x / \xi$, and $H_{n}(x)$ are the Hermite polynomials. Note however that, although the spatial dependence of the one-particle eigenfunctions is decoupled along the various directions, fermion gases in different dimensions present notable differences due to the nontrivial filling of the lowest $N$ states which provides the ground state of the $N$-particle system.

The above one-particle eigensolutions allow us to reconstruct the corresponding fermion-gas ground state (11), and study its general properties by computing particle correlations and bipartite entanglement entropies. In the following we also set $l=1$, thus

$$
\xi \equiv l^{\theta}=1, \quad X \equiv x / \xi=x .
$$

The dependence on $\hbar, m$ and $l=\omega^{-1}$ of the quantities considered can be easily reconstructed by a dimensional analysis.

\section{B. Observables}

\section{One-particle and density correlations}

The one-particle correlation function reads

$$
C(\mathbf{x}, \mathbf{x}) \equiv\left\langle c^{\dagger}(\mathbf{x}) c(\mathbf{y})\right\rangle=\sum_{i=1}^{N} \psi_{i}(\mathbf{x})^{*} \psi_{i}(\mathbf{y})
$$

where $c(\mathbf{x})$ is the fermionic annihilation operator. The particle density and the connected density-density correlation are respectively given by

$$
\begin{aligned}
& \rho(\mathbf{x}) \equiv\langle n(\mathbf{x})\rangle=C(\mathbf{x}, \mathbf{x})=\sum_{i=1}^{N}\left|\psi_{i}(\mathbf{x})\right|^{2} \\
& G_{n}(\mathbf{x}, \mathbf{y}) \equiv\langle n(\mathbf{x}) n(\mathbf{y})\rangle_{c}= \\
& \quad=-|C(\mathbf{x}, \mathbf{y})|^{2}+\delta(\mathbf{x}-\mathbf{y}) C(\mathbf{x}, \mathbf{y})
\end{aligned}
$$

where $n(\mathbf{x})=c(\mathbf{x})^{\dagger} c(\mathbf{x})$ is the particle-density operator, and we used the Wick theorem to write $G_{n}$ in terms of the two-point function $C$.

\section{Particle fluctuations and entanglement entropies}

Other important measures of the quantum correlations are related to extended spatial regions, such as the distribution of the particle number and the entanglement with the rest of the system.

The expectation value and connected correlators

$$
N_{A}=\left\langle\hat{N}_{A}\right\rangle, \quad\left\langle\hat{N}_{A}^{m}\right\rangle_{c}=\int_{A} \prod_{i=1}^{m} d^{d} x_{i}\left\langle\prod_{i=1}^{m} n\left(\mathbf{x}_{i}\right)\right\rangle_{c}
$$

of the particle-number operator of an extended region $A$,

$$
\hat{N}_{A}=\int_{A} d^{d} x n(\mathbf{x})
$$

characterize the particle distribution within $A$. For this purpose, it is convenient to introduce the cumulants of the particle distribution, which can be defined through a generator function as [21]

$$
V_{A}^{(m)}=\left.\left(-i \partial_{\lambda}\right)^{m} \ln \left\langle e^{i \lambda \hat{N}_{A}}\right\rangle\right|_{\lambda=0}
$$

In particular, the particle variance reads

$$
V_{A} \equiv V_{A}^{(2)}=\left\langle N_{A}^{2}\right\rangle_{c} \equiv\left\langle N_{A}^{2}\right\rangle-\left\langle N_{A}\right\rangle^{2}
$$

(the superscript $m=2$ will be understood in the case of the particle variance). A measure of the entanglement of the extended region $A$ with the rest of the system is provided by the Rényi entanglement entropies, defined as

$$
S_{A}^{(\alpha)}=\frac{1}{1-\alpha} \ln \operatorname{Tr} \rho_{A}^{\alpha}
$$


where $\rho_{A}$ is the reduced density matrix $\rho_{A}$ of the subsystem $A$. For $\alpha \rightarrow 1$, we recover the $\mathrm{vN}$ definition

$$
S_{A} \equiv S_{A}^{(1)} \equiv-\operatorname{Tr} \rho_{A} \ln \rho_{A}
$$

(the superscript $\alpha=1$ will be understood in the case of the $\mathrm{vN}$ entanglement entropy).

In noninteracting Fermi gases the particle cumulants and the entanglement entropies of a subsystem $A$ can be related to the two-point function $C(x, y)$ restricted within $A$, which we denote by $\mathbb{C}_{A}(x, y)$. The particle number and cumulants within $A$ can be derived using the relations (see e.g. Ref. [18])

$$
\begin{aligned}
& N_{A}=\operatorname{Tr} \mathbb{C}_{A}, \\
& V_{A}^{(m)}=\left.\left(-i \partial_{z}\right)^{m} \mathcal{G}\left(z, \mathbb{C}_{A}\right)\right|_{z=0}, \\
& \mathcal{G}(z, \mathbb{X})=\operatorname{Tr} \ln \left[1+\left(e^{i z}-1\right) \mathbb{X}\right] .
\end{aligned}
$$

The $\mathrm{vN}$ and Rényi entanglement entropies can be evaluated from the eigevalues of $\mathbb{C}_{A}(x, y)$ (see Refs. 22, 23] for applications to lattice systems).

The computation of particle cumulants and entanglement entropies in Fermi gases of $N$ particles is much simplified by introducing the $N \times N$ overlap matrix $\mathbb{A}[24,25]$,

$$
\mathbb{A}_{n m}=\int_{A} d^{d} z \psi_{n}^{*}(z) \psi_{m}(z), \quad n, m=1, \ldots, N,
$$

where the integration is over the spatial region $A$, and involves the lowest $N$ energy levels. The overlap matrix $\mathbb{A}$ and the restricted two-point function $\mathbb{C}_{A}$ satisfy

$$
\operatorname{Tr} \mathbb{C}_{A}^{k}=\operatorname{Tr} \mathbb{A}^{k} \quad \forall k \in \mathbb{N},
$$

which implies that the particle cumulants and the entanglement entropies can be computed form the eigenvalues of the $N \times N$ overlap matrix $\mathbb{A}$. The eigenvalues $a_{i}$ of $\mathbb{A}$ are real and limited, $a_{i} \in(0,1)$.

The particle number and cumulants can be computed using the relations [19] $N_{A}=\operatorname{Tr} \mathbb{A}$ and

$$
V_{A}^{(m)}=\left.\left(-i \partial_{z}\right)^{m} \mathcal{G}(z, \mathbb{A})\right|_{z=0} .
$$

In particular,

$$
\begin{aligned}
& V_{A}=\operatorname{Tr} \mathbb{A}(1-\mathbb{A}), \\
& V_{A}^{(3)}=\operatorname{Tr}\left[\mathbb{A}-3 \mathbb{A}^{2}+2 \mathbb{A}^{3}\right], \\
& V_{A}^{(4)}=\operatorname{Tr}\left[\mathbb{A}-7 \mathbb{A}^{2}+12 \mathbb{A}^{3}-6 \mathbb{A}^{4}\right],
\end{aligned}
$$

etc.... The vN and Rényi entanglement entropies are obtained by [24, 26]

$$
S_{A}^{(\alpha)}=\sum_{n=1}^{N} s_{\alpha}\left(a_{n}\right),
$$

where $a_{n}$ are the eigenvalues of $\mathbb{A}$, and

$$
s_{\alpha}(\lambda)=\frac{1}{1-\alpha} \ln \left[\lambda^{\alpha}+(1-\lambda)^{\alpha}\right] .
$$

and, in particular,

$$
s_{1}(\lambda)=-\lambda \ln \lambda-(1-\lambda) \ln (1-\lambda)
$$

for the vN entropy. We also mention that, while $\operatorname{Tr} \mathbb{A}$ gives the average particle number $N_{A}$ within $A$, $\operatorname{det} \mathbb{A}$ is the probability to find all particles within $A$.

We consider two different partitions of the space:

(i) The subsystem $B$ is separated from the rest by a hyperplane at a distance $x$ from the center of the trap. The corresponding $x$-dependent particle cumulants and entanglement entropies are denoted by $V_{B}^{(m)}(x)$ and $S_{B}^{(\alpha)}(x)$. In one dimension, the subsystem $B$ is given by the infinite interval $B=[-\infty, x]$. The half-space quantities are defined as

$$
V_{\mathrm{HS}}^{(m)} \equiv V_{B}^{(m)}(0), \quad S_{\mathrm{HS}}^{(\alpha)} \equiv S_{B}^{(\alpha)}(0) .
$$

We also define

$$
S_{\Delta}^{(\alpha)}(x) \equiv S_{B}^{(\alpha)}(x)-S_{B}^{(\alpha)}(0) .
$$

(ii) The subsystem $S$ is a region containing the center of trap, and enclosed by two parallel hyperplanes at distance $x$ from the center. The corresponding entanglement entropies are denoted by $V_{S}^{(m)}(x)$ and $S_{S}^{(\alpha)}(x)$. In one dimension, the subsystem $S$ is given by the symmetric interval $S=[-x, x]$ (where $x=0$ corresponds to the center of the trap).

\section{FERMI GASES IN 1D TRAPS}

In this section we consider 1D noninteracting spinless fermion gases of $N$ particles confined by a power-law external potential, in particular by a harmonic potential. This model has a wider application, because 1D Bose gases in the limit of strong short-ranged repulsive interactions can be mapped into a spinless fermion gas. The basic model to describe the many-body features of a boson gas confined to an effective 1D geometry is the Lieb-Liniger model with an effective two-particle repulsive contact interaction [27],

$$
\mathcal{H}_{\mathrm{LL}}=\sum_{i=1}^{N}\left[\frac{p_{i}^{2}}{2 m}+V\left(x_{i}\right)\right]+g \sum_{i \neq j} \delta\left(x_{i}-x_{j}\right)
$$

where $N$ is the number of particles and $V(x)$ is the confining potential. The limit of infinitely strong repulsive interactions corresponds to a $1 \mathrm{D}$ gas of impenetrable bosons [28], the Tonks-Girardeau gas. 1D Bose gases with repulsive two-particle short-ranged interactions become more and more nonideal with decreasing the particle density, acquiring fermion-like properties, so that the 1D gas of impenetrable bosons is expected to provide an effective description of the low-density regime of confined 1D bosonic gases 29]. Therefore, due to the mapping between 1D gases of impenetrable bosons and 
spinless fermions, some correlations in free fermion gases are identical to those of the hard-core boson gases, such as those related to the particle density, particle fluctuations of extended regions, and bipartite entanglement entropies of connected parts. This correspondence holds also in the presence of an external space-dependent potential.

\section{A. The particle correlators}

In 1D noninteracting Fermi systems with $N$ particles in a harmonic trap, the two-point correlation function (10) can be written as

$$
C(x, y)=\frac{N^{1 / 2}}{\sqrt{2}} \frac{\phi_{N+1}(x) \phi_{N}(y)-\phi_{N}(x) \phi_{N+1}(y)}{x-y},
$$

where we used the Christoffel-Darboux relation for othornormal polynomials. The particle density $\rho(x)=$ $C(x, x)$,

$$
\rho(x)=\frac{N^{1 / 2}}{\sqrt{2}}\left[\phi_{N+1}^{\prime}(x) \phi_{N}(x)-\phi_{N}^{\prime}(x) \phi_{N+1}(x)\right],
$$

shows a peculiar behavior characterized by $N$ local maxima, which get suppressed by powers of $1 / N$ with increas$\operatorname{ing} N$.

Since the particle density and the density correlator of free fermion gases are equal to those of boson gases in the hard-core limit, the results already obtained for system of impenetrable bosons in a trapping potential apply also to trapped fermion gases. The large- $N$ asymptotic expansion is known to $O(1 / N)$ [30, 31]. The leading behavior is given by

$$
\rho(x)=N^{1 / 2}\left[R_{\rho}(\zeta)+O(1 / N)\right], \quad \zeta \equiv x / N^{1 / 2},
$$

with

$$
R_{\rho}(\zeta)=\frac{1}{\pi} \sqrt{2-\zeta^{2}}, \quad \zeta \leq \zeta_{c}=\sqrt{2}
$$

and $R_{\rho}(\zeta)=0$ for $\zeta>\zeta_{c}=\sqrt{2}$.

The space dependence of the connected correlation function of the particle density operator $n_{x}=c(x)^{\dagger} c(x)$ presents a different large- $N$ scaling behavior, characterized by different power laws [32], i.e.,

$$
G(x, y) \approx N R_{G}\left(N^{1 / 2} x, N^{1 / 2} y\right),
$$

for $x \neq y$, as shown by Fig. 11. Note that the asymptotic regime of $G_{n}$ is not approached uniformly when $x \rightarrow y$, because $G(x, x)=\rho(x)-\rho(x)^{2}$, cf. Eq. (12).

The large- $N$ scaling function of the particle density in a harmonic trap vanishes at $\zeta_{c}=\sqrt{2}$ where $R_{\rho}\left(\zeta_{c}\right)=0$. Around this point the particle correlations [32], and also the entanglement entropies, show a different large- $N$ scaling behaviors characterized by other power laws 33]. Indeed, around the point $x_{c} \equiv N^{1 / 2} \zeta_{c}$, the particle density

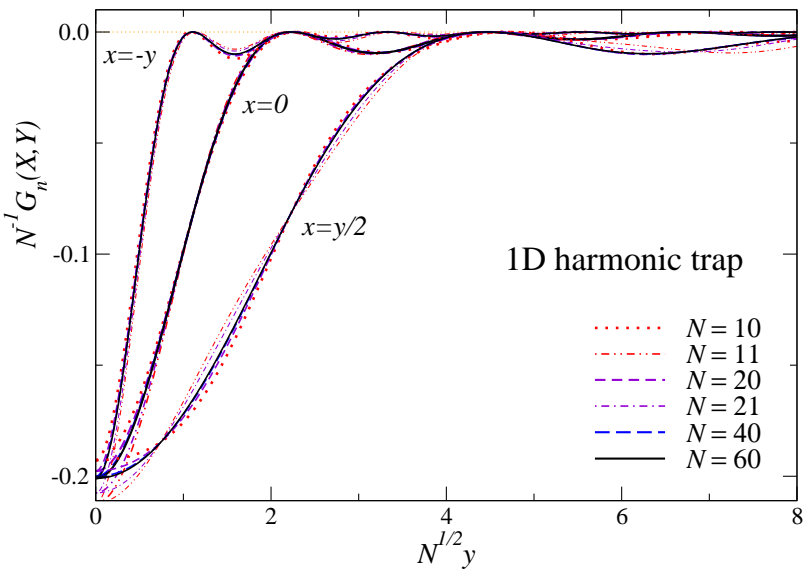

FIG. 1: (Color online) Large- $N$ scaling of $G_{n}(x, y)$ for $x=$ $y / 2, x=0$, and $x=-y$ for $1 \mathrm{D}$ fermion gases in a harmonic trap. We plot $N^{-1} G_{n}(x, y)$ vs $N^{1 / 2} y$.

and its correlation behave as

$$
\begin{aligned}
& \rho(x) \approx N^{1 / 6} g_{\rho}\left[N^{1 / 6}\left(x-x_{c}\right)\right], \quad x_{c}=N^{1 / 2} \zeta_{c}, \\
& G_{n}\left(x_{c}, x\right) \approx N^{1 / 3} g_{n}\left[N^{1 / 6}\left(x-x_{c}\right)\right] .
\end{aligned}
$$

The scaling function $g_{\rho}(z)$ can be obtained from related computations within the Gaussian unitary ensembles of random matrices [31, 34]:

$$
\begin{aligned}
g_{\rho}(z) & =\operatorname{Lim}_{N \rightarrow \infty} N^{-1 / 6} \rho\left[N^{1 / 2}\left(\zeta_{c}+N^{-2 / 3} z\right)\right] \\
& =2^{1 / 2}\left|\operatorname{Ai}^{\prime}\left(2^{1 / 2} z\right)\right|^{2}-2 z\left|\operatorname{Ai}\left(2^{1 / 2} z\right)\right|^{2}
\end{aligned}
$$

\section{B. Spatial entanglement}

\section{Half-space entanglement entropy}

The asymptotic large- $N$ behavior of the half-space ( $A=[-\infty, 0]$ where $x=0$ is the center of the trap) vN and Rényi entanglement entropies can be inferred by exploiting known results for the $1 \mathrm{D}$ hard-core BoseHubbard model in the presence of an external power-law potential $V(x)=(x / l)^{p}$ and a chemical potential, which is equivalent to a lattice free-fermion model. The derivation is outlined in App. A. We obtain

$$
\begin{aligned}
& S_{\mathrm{HS}}^{(\alpha)}=S_{\mathrm{ASY}}^{(\alpha)}+o\left(N^{0}\right), \\
& S_{\mathrm{ASY}}^{(\alpha)}=C_{\alpha}\left[\ln N+\ln \frac{4(p+2)}{p}+y_{\alpha}\right],
\end{aligned}
$$

where

$$
C_{\alpha}=\frac{1+\alpha^{-1}}{12}
$$

$p$ is the power-law of the potential, and $y_{\alpha}$ is given in Eq. A12). Note that the leading logarithmic term, and in particular its coefficient, is independent of the trapping 


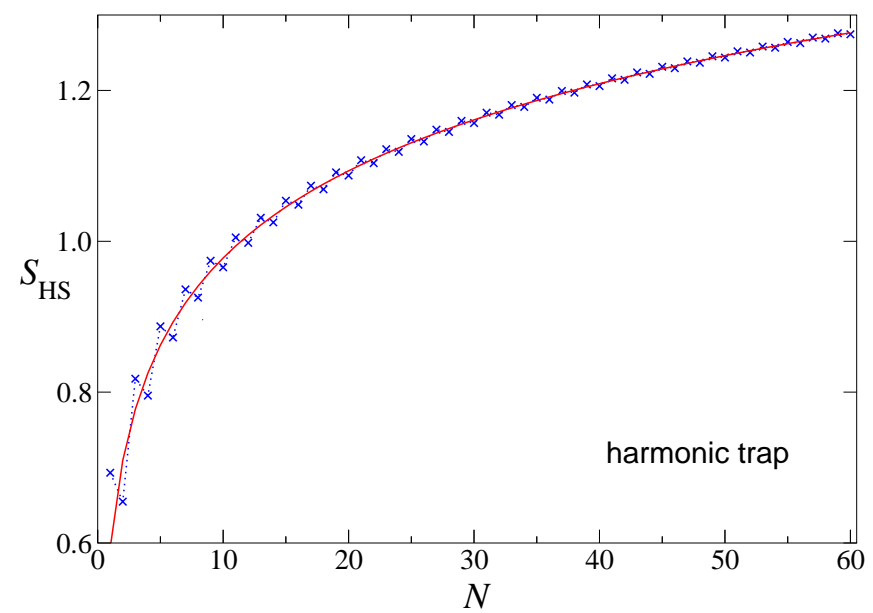

FIG. 2: (Color online) The half-space vN entanglement entropy. The full line shows the large- $N$ asymptotic behavior (42).
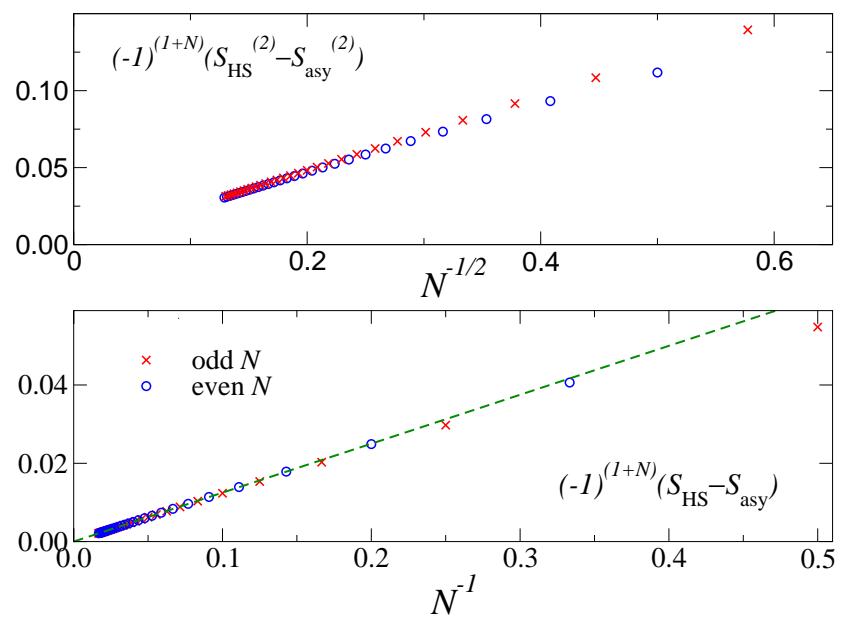

FIG. 3: (Color online) We plot $(-1)^{1+N}\left(S_{\mathrm{HS}}-S_{\mathrm{ASY}}\right)$ vs $N^{-1}$ (bottom) and $(-1)^{1+N}\left(S_{\mathrm{HS}}^{(2)}-S_{\mathrm{ASY}}^{(2)}\right)$ vs $N^{-1 / 2}$ (top). In the bottom figure the dashed line shows the slope $1 / 8$.

potential, and it is equal to that of homogeneous systems with open boundary conditions [24, 26], which is determined by the corresponding conformal field theory 35] with central charge $c=1$. The asymptotic behavior (42) in the limit $p \rightarrow \infty$ reproduces the results for homogeneous systems with open boundary conditions [24, 26]

$$
S_{\mathrm{HS}}^{(\alpha)}=C_{\alpha}\left[\ln N+\ln 4+y_{\alpha}+O\left(N^{-1 / \alpha}\right)\right],
$$

because in the limit $p \rightarrow \infty$ the system becomes equivalent to a Fermi gas confined by a 1D hard-wall trap of size $L=2 l$.

In order to check the convergence to this asymptotic behavior, we numerically compute the half-space entanglement entropies $S_{\mathrm{HS}}^{(\alpha)}$ of $N$ particles in the presence of a harmonic trap. We use the method based on the overlap matrix (22), i.e. we numerically compute its eigenvalues and then obtain the entanglement entropies through Eq. (28). Figs. 2 and 3 show data for the vN and $\alpha=2$ Rényi entropies. They are fully consistent with the asymptotic behavior (42). In particular, the large- $N$ behavior of the $\mathrm{vN}$ entropy turns out to behave as

$$
S_{\mathrm{HS}}=S_{\mathrm{ASY}}+(-1)^{N} \frac{c_{1}}{N}+\frac{c_{2}}{N^{2}}+(-1)^{N} \frac{c_{3}}{N^{3}}+\ldots
$$

with $c_{1} \approx-1 / 8$ (with a precision better than $10^{-6}$, see Fig. 3, $c_{2} \approx 0.009893$ and $c_{3} \approx 0.066$ (the uncertainty should be on the last figures). The data of the $\alpha=2$ Rényi entropy, shown in Fig. 3. fits the Ansatz

$$
S_{\mathrm{HS}}^{(2)}=S_{\mathrm{ASY}}^{(2)}+(-1)^{N} \frac{b_{1}}{N^{1 / 2}}+\frac{b_{2}}{N}+\ldots
$$

with $b_{1} \approx-0.2387$ and $b_{2} \approx 0.0146$. Note that the above numerical results show that the corrections to the large$N$ asymptotic behavior are $O\left(N^{-1 / \alpha}\right)$ in Eq. (41), analogously to homogeneous systems, cf. Eq. (44).

Finally, we mention that the effects of a power-law trapping potential on the scaling behavior of the entanglement at the quantum critical point of $1 \mathrm{D}$ lattice models were investigated in Refs. [33, 36-38]. In particular, the 1D hard-core Bose-Hubbard model, which is equivalent to a free fermion lattice model, was considered in the superfluid phase at half filling [33]. As shown by the arguments reported in App. A, used to derive Eq. (42), these results are somehow related with the large- $N$ scaling of 1D Fermi gases investigated in this section, in particular when the chemical potential is driven toward the superfluid-to-empty transition. However the large- $N$ scaling behavior of 1D continuum Fermi gases presents distinct features, as pointed out in Refs. 24, 26] in the case of homogenous systems.

\section{Finite intervals around the center of the trap}

We now consider a symmetric interval $S=[-x, x]$ around the center of the trap. By integrating the large- $N$ particle density (35) within the interval $S=[-x, x]$, we obtain the average number $N_{S}(x)$ of particles within $S$ in the large- $N$ limit,

$$
\frac{N_{S}(x)}{N}=\frac{1}{\pi}\left[\zeta \sqrt{2-\zeta^{2}}+2 \arcsin (\zeta / \sqrt{2})\right]+O(1 / N)
$$

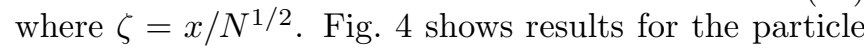
number within $S$ at fixed $N$, up to $N=100$. They show that the large- $N$ limit (47) is rapidly approached by the data.

Results for the $\mathrm{vN}$ and $\alpha=2$ entanglement entropies up to $N=180$ are shown in Figs. 5 . With increasing $N$, the subtracted data of $S_{S}^{(\alpha)}-2 C_{\alpha} \ln N$ appear to approach a function of $\zeta \equiv x / N^{1 / 2}$. Therefore, we infer the large- $N$ scaling behavior

$$
S_{S}^{(\alpha)}(x) \approx 2 C_{\alpha}\left[\ln N+y_{\alpha}+\ln 4+f_{S}^{(\alpha)}(\zeta)\right]
$$




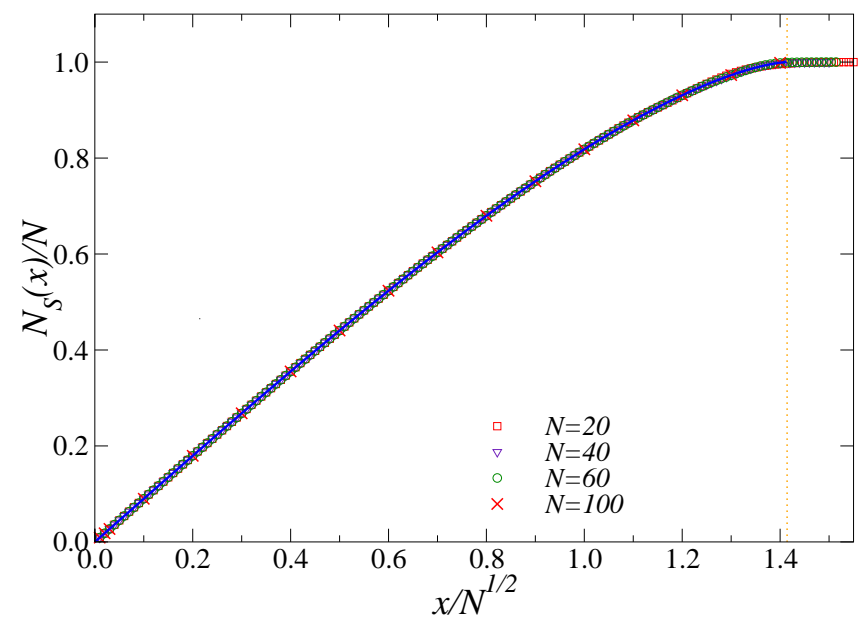

FIG. 4: (Color online) The particle number within the interval $S=[-x, x]$ around the center of the trap, for some values of $N$ up to $N=100$, versus $\zeta \equiv x / N^{1 / 2}$. The full line (hardly visible among the data symbols) shows the large- $N$ limit (47) of the ratio $N_{S}(x) / N$.

The scaling functions $f_{S}^{(\alpha)}(\zeta)$ are expected to be singular at $\zeta=0$ corresponding to a vanishing interval, and at $\zeta=$ $\sqrt{2}$, which corresponds to the point where the particle density vanishes in the large- $N$ limit, cf. Eq. (35). Note that the space dependence scales analogously to that of the particle density, cf. Eq. (35), while it differs from that of the connected density-density correlation, cf Eq. (37).

The large- $N$ convergence is rapid at least up to $\zeta \approx 1$, but also the data for $1 \lesssim \zeta \lesssim \sqrt{2}$ appear to approach a unique curve, although more slowly. Moreover, the behavior of the data with increasing $N$ suggests that the large- $N$ scaling functions $f_{S}^{(\alpha)}(\zeta)$ are independent of $\alpha$. Actually, they turn out to be well approximated by the simple function

$$
f_{S}^{(\alpha)}(\zeta) \approx f_{a}(\zeta)=\ln \sin (\pi \zeta / \sqrt{2})+\ln (2 / \pi),
$$

as shown in Fig. 5 for the $\alpha=1 \mathrm{vN}$ and $\alpha=2$ Rényi entropies. In Fig. 6 we show the differences between the vN entropy $S_{S}(x)$ and the asymptotic behavior (48) with $f_{S}$ given by Eq. (49), at its maximum $\zeta=2^{-1 / 2}$ and at $\zeta=2^{-3 / 2}$. For example at $\zeta=2^{-1 / 2}$ the data show deviations smaller than 0.01 for $N \gtrsim 100$, suggesting that the deviation in the large- $N$ limit should be less than 0.01. Smaller deviations are observed at $\zeta=2^{-3 / 2}$, see Fig. 6. A more precise large- $N$ extrapolation is made difficult by the presence of oscillations, whose structure is not clear, see Fig. 6.

It is worth comparing the above results with the behavior of analogous quantities in homogeneous Fermi gas within hard walls, whose entanglement entropies of the interval $S=[-x, x]$ around the center of the hard-wall trap of size $L=2 l=2$ are given by [26]

$S_{S}^{(\alpha)}(x) \approx 2 C_{\alpha}\left[\ln N+\ln \sin (\pi x)+y_{\alpha}+\ln 2+O\left(N^{-1 / \alpha}\right)\right]$
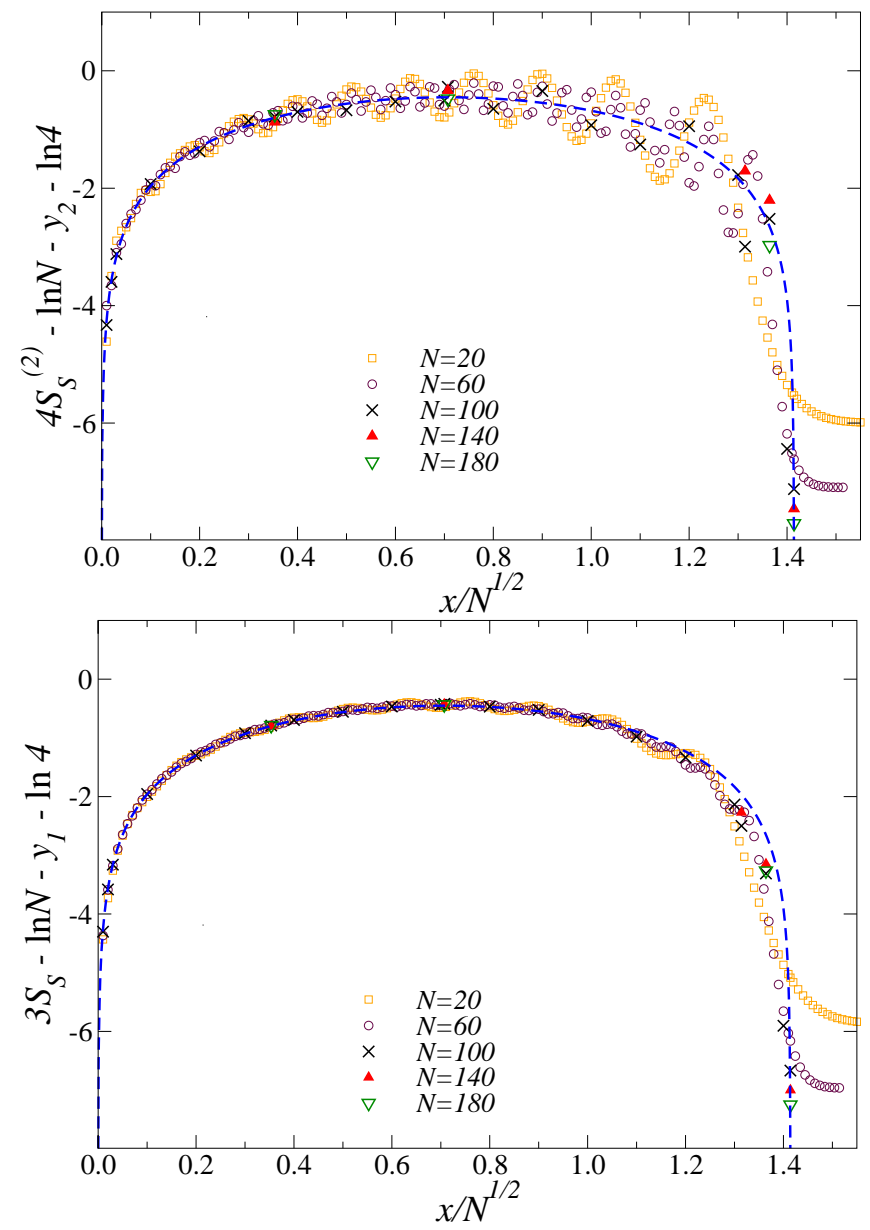

FIG. 5: (Color online) The $\alpha=1 \mathrm{vN}$ (bottom) and $\alpha=2$ Rényi (top) entanglement entropies of the interval $S \equiv[-x, x]$ vesus $\zeta \equiv x / N^{1 / 2}$. We plot $S_{S}^{(\alpha)}(x) /\left(2 C_{\alpha}\right)-\left(\ln N+y_{\alpha}+\ln 4\right)$ for some values of $N$ up to $N=180$. The full lines show the function (49).
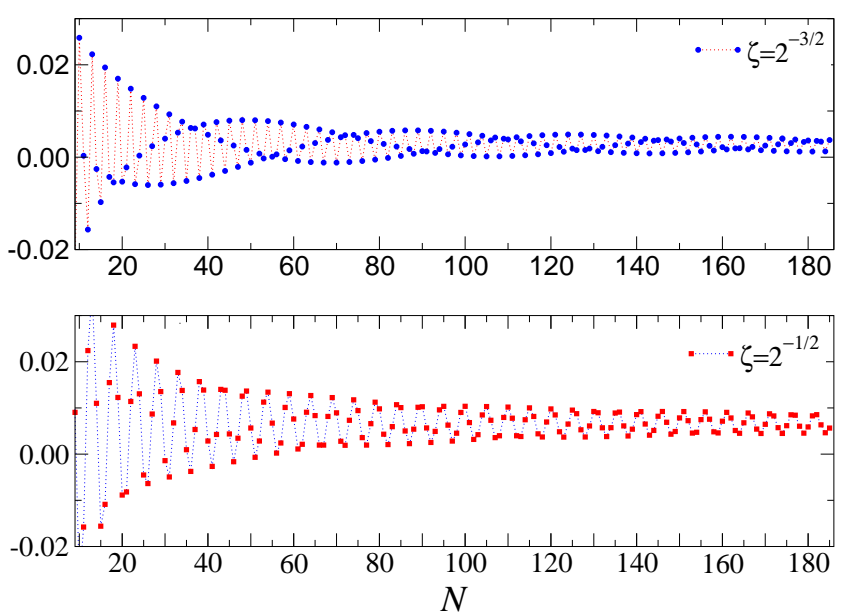

FIG. 6: (Color online) Differences between $S_{S}(x)$ and the asymptotic behavior (48) with $f_{S}$ given by Eq. (49) at $\zeta=$ $2^{-1 / 2}$, which is the maximum of Eq. (49), and $\zeta=2^{-3 / 2}$, versus $N$. 

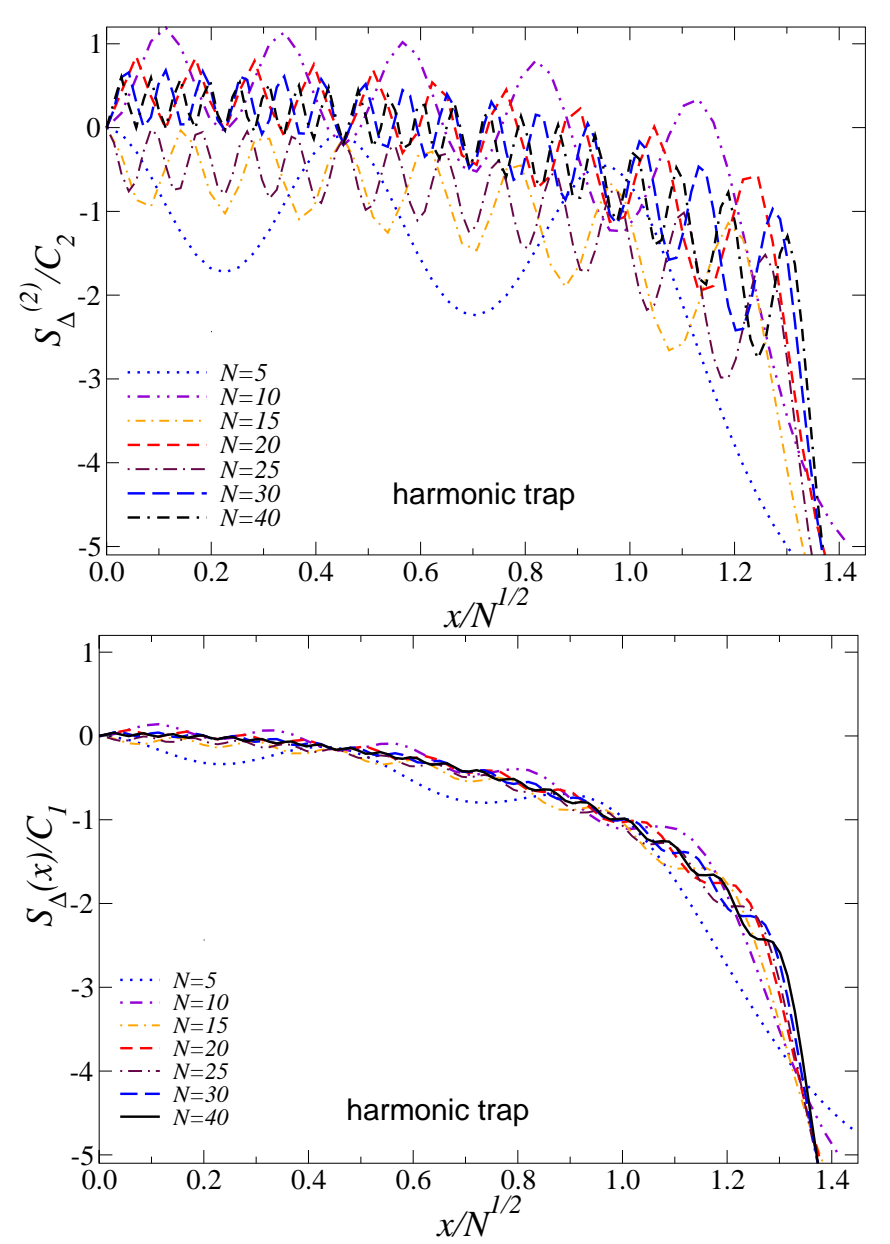

FIG. 7: (Color online) $S_{\Delta}^{(\alpha)}(x) / C_{\alpha}$ versus $x / N^{1 / 2}$ for the $\alpha=$ $1 \mathrm{vN}$ (bottom) and $\alpha=2$ (top) entropies, and several values of $N$. The two sets of data appear to approach the same large- $N$ limit.

Finally, Fig. 7 shows results for the quantity $S_{\Delta}^{\alpha}(x) \equiv$ $S_{B}^{\alpha}(x)-S_{\mathrm{HS}}^{\alpha}$, i.e. the difference between the entanglement entropies of the intervals $[-\infty, x]$ and $[-\infty, 0]$. They show the large- $N$ scaling behavior

$$
S_{\Delta}^{(\alpha)}(x) \approx C_{\alpha} f_{\Delta}^{(\alpha)}(\zeta), \quad \zeta \equiv x / N^{1 / 2}
$$

They also suggest that $f_{\Delta}^{(1)}=f_{\Delta}^{(2)}$, i.e. $f_{\Delta}^{(\alpha)}$ is independent of $\alpha$, although the convergence of the $\alpha=2$ Rényi entropy is slower than that of the $\mathrm{vN}$ entropy, apparently $O\left(N^{-1 / 2}\right)$ against $O\left(N^{-1}\right)$.

\section{Particle fluctuations in extended spatial subsystems}

Some results for the half-space particle variance and quartic cumulant are shown in Fig. 8. They are characterized by large odd-even oscillations in the number of particles. An educated guess for the asymptotic large- $N$ behavior of the half-space particle variance in a generic

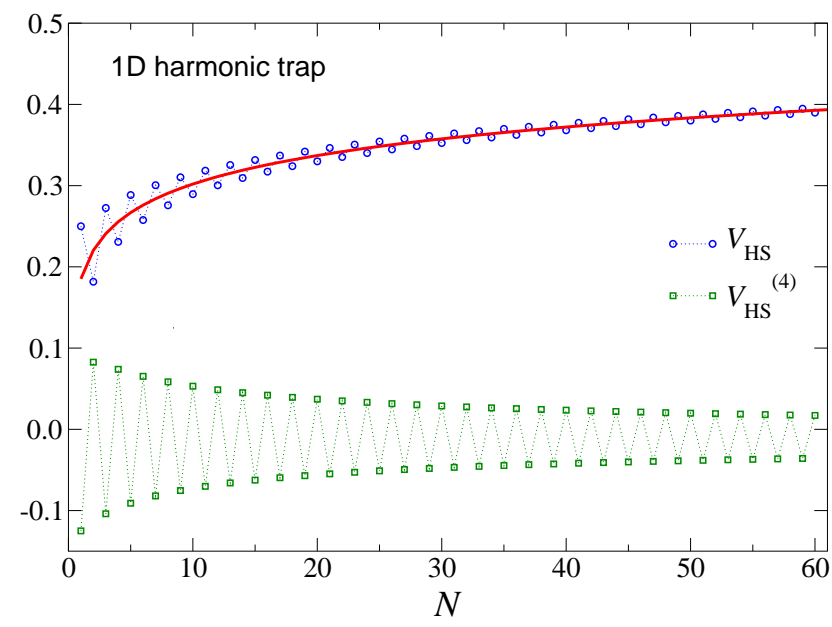

FIG. 8: (Color online) The half-space particle-number cumulants $V_{\mathrm{HS}}$ and $V_{\mathrm{HS}}^{(4)}$. The full line shows the function (52).
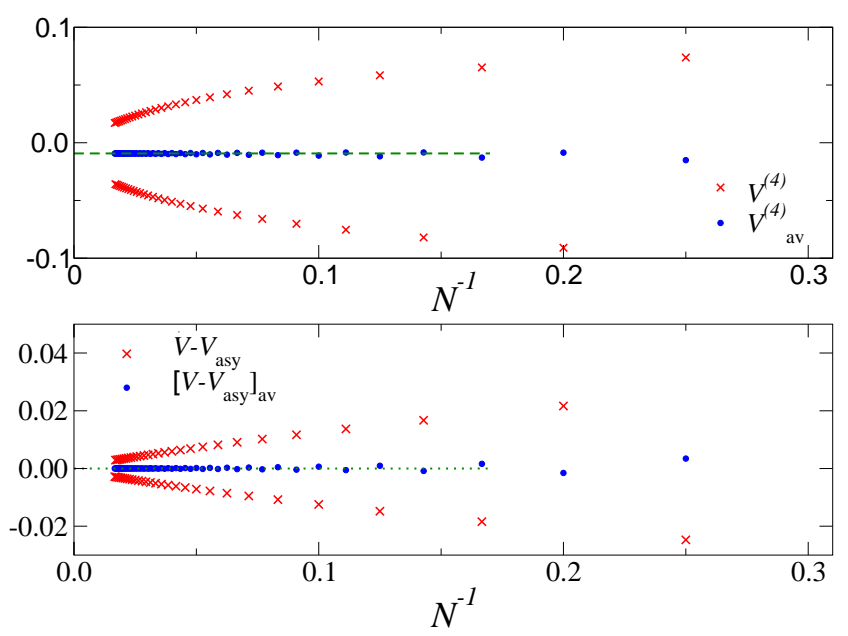

FIG. 9: (Color online) Check of the asymptotic behavior for the particle variance and quartic cumulant of half space. The subscript "av" indicates the average over the last two data to suppress the large oscillations. The dotted and dashed lines show the $N \rightarrow \infty$ limit expected for $V_{\mathrm{HS}}-V_{\mathrm{ASY}} \approx 0$ and $V_{\mathrm{HS}}^{(4)} \approx-0.009255$.

external potential $V(x) \propto(x / l)^{p}$ is

$$
\begin{aligned}
& V_{\mathrm{HS}} \approx V_{\mathrm{ASY}}+o\left(N^{0}\right), \\
& V_{\mathrm{ASY}}=\frac{1}{2 \pi^{2}}\left[\ln N+\ln \frac{4(p+2)}{p}+1+\gamma_{E}\right] .
\end{aligned}
$$

This asymptotic behavior is somehow derived by analogy with the asymptotic behavior of the half-space Rényi entanglement entropies, taking also into account the known asymptotic behavior of the particle variance in hard-wall traps [19],

$$
V_{\mathrm{HS}}=\frac{1}{2 \pi^{2}}\left[\ln N+\ln 4+1+\gamma_{E}+O\left(N^{-1}\right)\right],
$$

which must be recovered in $p \rightarrow \infty$ limit. 
Concerning the other cumulants, we expect that the leading term is the same as that of homogenous systems within hard walls, like the leading terms of the entanglement entropies and particle variance. Thus

$$
V_{\mathrm{HS}}^{(2 i)}=\nu_{2 i}+o\left(N^{0}\right) \text { for } i>2
$$

where $v_{2 i}$ are the same constant appearing in the case of the hard-wall trap [19], i.e. $\nu_{4}=-0.0092552, \nu_{6}=$ 0.00404469 , etc...

The above large- $N$ predictions are fully supported by the numerical data at fixed $N$ with increasing $N$, as shown in Fig. 9] where we also show data averaged over two subsequent particle numbers to suppress the oddeven oscillations. In the case of the particle variance, the amplitude of the odd-even oscillations appear to decrease as $O(1 / N)$, while the average between the data for subsequent particle numbers approaches the predicted asymptotic behavior much more rapidly. In the case of the quartic cumulant, the oscillations get suppressed more slowly, but their odd-even average approaches the predicted value quite rapidly. For the largest available values of $N$ the difference of these averages from the asymptotic predicted behaviors is $O\left(10^{-5}\right)$.

We now consider the interval $S=[-x, x]$ around the center of the trap. In Fig. 10 we show the particle variance for values of $N$ up to $N=180$. They show a behavior analogous to that of the entanglement entropies, see Fig. 5, and are consistent with

$$
V_{S}(x) \approx \frac{1}{\pi^{2}}\left[\ln N+1+\gamma_{E}+\ln 4+f_{V}(\zeta)\right]
$$

The analysis of the data with increasing $N$ is consistent with the relation $f_{V}(\zeta)=f_{S}^{(\alpha)}(\zeta)$, where $f_{S}^{(\alpha)}(\zeta)$ are the corresponding scaling functions of the entangelement entropies, cf. Eq. (48). Therefore, $f_{V}(\zeta)$ is well approximated by the same function $f_{a}(\zeta)$, cf. Eq. (49). Again, this behavior resembles that of the homogeneous system within a hard-wall trap of size $L=2 l=2$, which is [19]

$$
\begin{aligned}
V_{S}(x)= & \frac{1}{\pi^{2}}[\ln N+\ln \sin (\pi x)+ \\
& \left.+1+\gamma_{E}+\ln 2+O\left(N^{-1}\right)\right]
\end{aligned}
$$

In Fig. 11 we show the third and quartic cumulants of the interval $S=[-x, x]$. They are characterized by oscillations which increase when $\zeta \rightarrow \sqrt{2}$, but remain apparently limited with increasing $N$.

\section{HIGHER-DIMENSIONAL SYSTEMS}

In this section we consider Fermi gases confined by twoand three-dimensional traps. We again study the large$N$ behavior of the particle correlators, cumulants of the particle-number distribution and entanglement entropies of extended spatial regions.

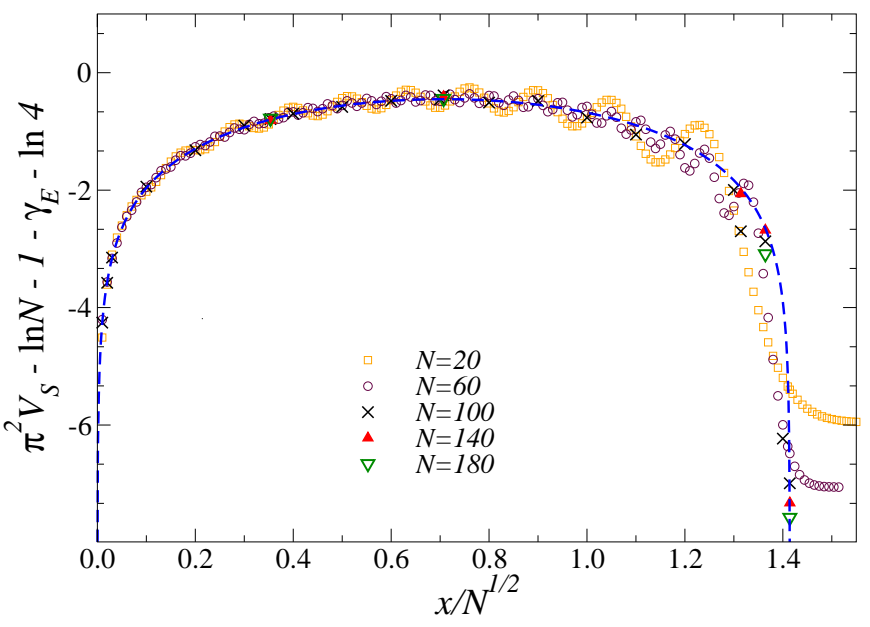

FIG. 10: (Color online) The particle variance of intervals $S=[-x, x]$ for some values of $N$, versus $\zeta=x / N^{1 / 2}$. We plot $\pi^{2} V-\left(\ln N+1+\gamma_{E}+\ln 4\right)$ The line shows the function (49).

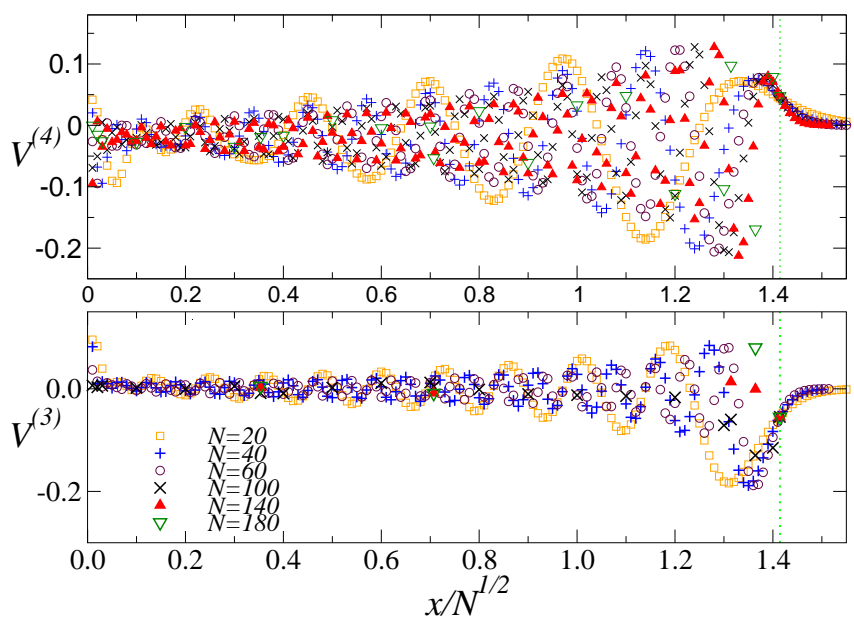

FIG. 11: (Color online) The third (top) and quartic (bottom) cumulants of the interval $S=[-x, x]$.

The $\mathrm{vN}$ and Rényi entanglement entropies of extended spatial subsystems in the ground state of homogenous Fermi gases of $d$ dimension grow asymptotically as $N^{(d-1) / d} \ln N$, with a prefactor that is analytically computed using the Widom conjecture [39], for both periodic and open boundary conditions. The logarithmic correction to the power-law behavior is related to the area-law violation in lattice free fermions [40 47], i.e. for a large subsystem $A$ of linear size $\ell$ in an infinite $d$ dimensional lattice the entanglement entropies scale like $S^{(\alpha)}(A) \sim \ell^{d-1} \ln \ell$. In this section we study the effects of a space-dependent confining potential in $2 \mathrm{D}$ and $3 \mathrm{D}$ Fermi systems, investigating again the relations between particle fluctuations and entanglement entropies. 

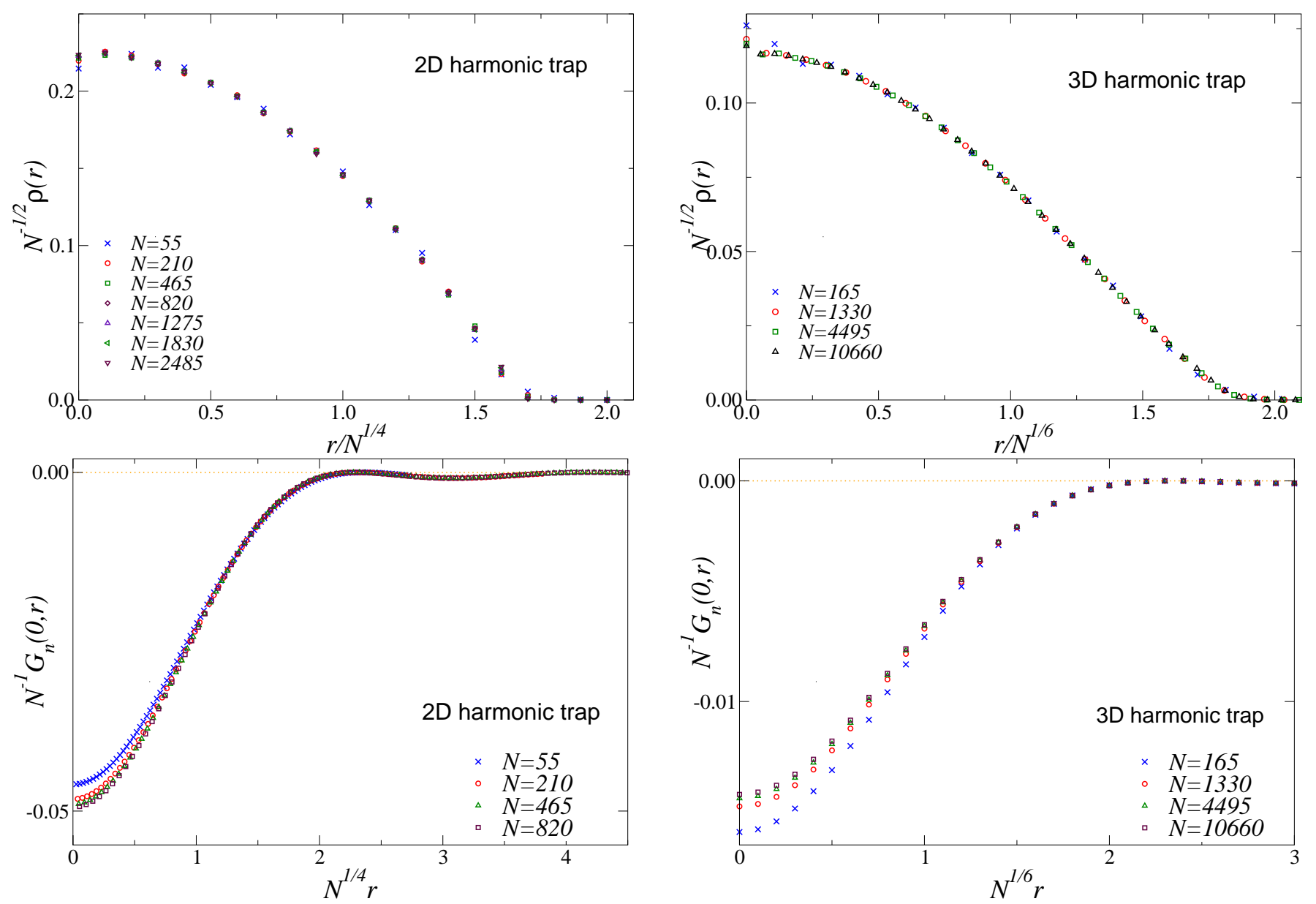

FIG. 12: (Color online) The particle density and the density correlator for $2 \mathrm{D}$ systems in a harmonic trap: $N^{-1 / 2} \rho(r)$ vs $r / N^{1 / 4}$ (top) and $N^{-1} G_{n}(0, \vec{x})$ vs $N^{1 / 4} r$ (bottom) where $r \equiv$ $|\vec{x}|$ is the distance from the center of the trap.

\section{A. Particle density and its correlator}

Using the results of Sec. II we can easily obtain results for the particle density and the density correlator in the presence of trap. Some data for $2 \mathrm{D}$ and $3 \mathrm{D}$ systems in a harmonic trap are shown in Figs. 12 and 13. They show the scaling behavior

$$
\begin{aligned}
& \rho(\mathbf{x}) \approx N^{1 / 2} R_{\rho}\left(r / N^{1 /(2 d)}\right), \\
& G_{n}(0, \mathbf{x}) \approx N R_{G}\left(0, r N^{1 /(2 d)}\right),
\end{aligned}
$$

where $r \equiv|\mathbf{x}|$. Note that, even in dimensions higher than one, the large- $N$ space rescalings of the particle density and its connected correlation are different.

\section{B. Half-space entanglement entropies and particle fluctuations}

In homogeneous systems with periodic and open boundary conditions, the half-space entaglement en-

FIG. 13: (Color online) The particle density and the density correlator for $3 \mathrm{D}$ systems in a harmonic trap: $N^{-1 / 2} \rho(r)$ vs $r / N^{1 / 6}$ (top) and $N^{-1} G_{n}(0, \vec{x})$ vs $N^{1 / 6} r$ (bottom) where $r \equiv$ $|\vec{x}|$ is the distance from the center of the trap.

tropies of a square $L^{2}$ system with open boundary conditions behave as 13 ]

$$
S_{\mathrm{HS}}^{(\alpha)} \approx c N^{1 / 2} \ln N, \quad c=\frac{1+\alpha^{-1}}{12 \pi^{1 / 2}}
$$

The asymptotic large- $N$ behavior of the half-space particle cumulants and Rényi entanglement entropies can be also computed analytically in the presence of an external harmonic potential. For this purpose, we exploit the fact that the corresponding overlap matrix (22) is a block diagonal matrix. Indeed, relabeling the indeces $n, m$ of the $N \times N$ overlap matrix as $n_{1}, \ldots, n_{d}$, using Eq. (5), we can write the half-space overlap matrix as

$$
\mathbb{A}_{n_{1}, \ldots, n_{d} ; m_{1}, \ldots, m_{d}}=\prod_{i=2}^{d} \delta_{n_{i} m_{i}} \int_{0}^{\infty} d z \phi_{n_{1}}(z) \phi_{m_{1}}(z)
$$

where $\phi_{n}$ are the 1D eigenfunctions (7), the indeces $n_{1}, \ldots, n_{d}$ correspond to the lowest $N$ states according to the Eqs. (6) and (8). 

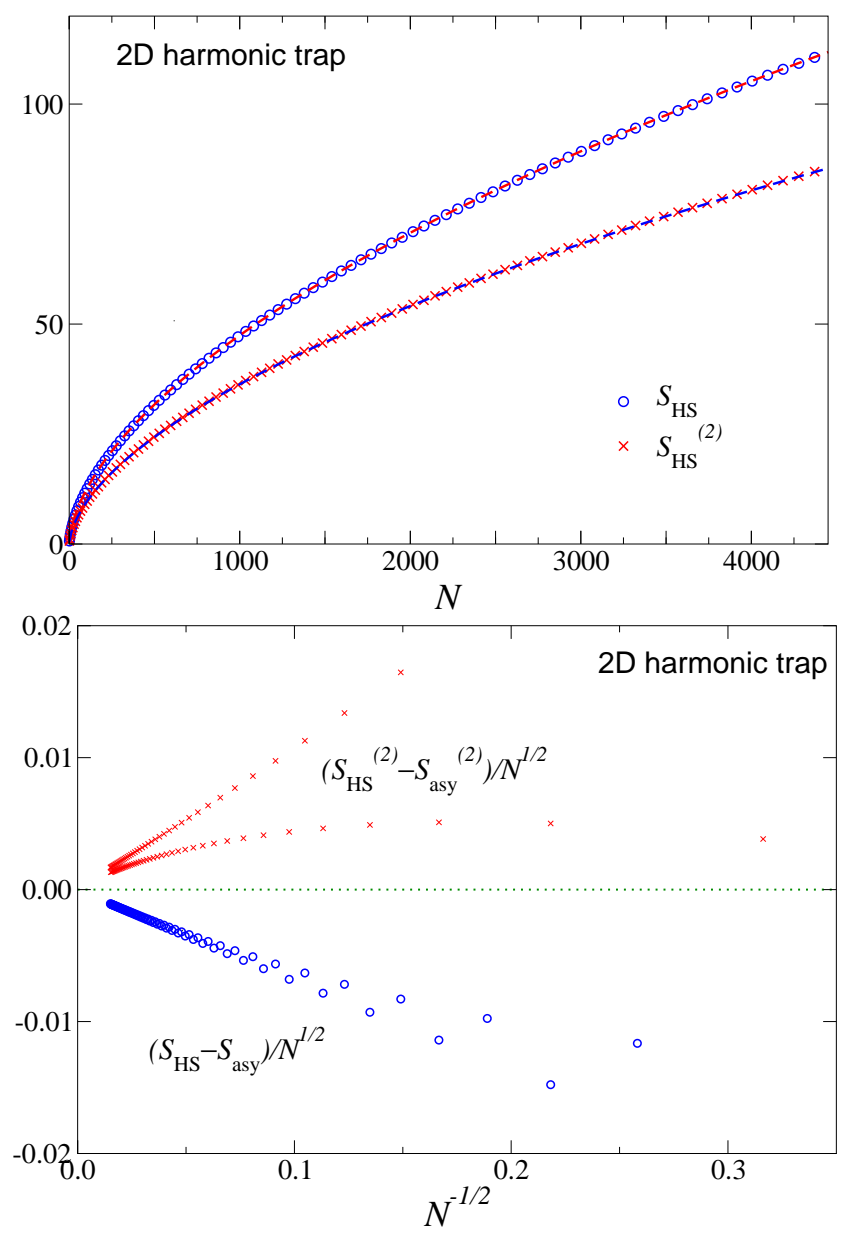

FIG. 14: (Color online) In the top figure we show the halfspace $\mathrm{vN}$ and $\alpha=2$ Rényi entanglement entropies of $2 \mathrm{D}$ systems with harmonic trap. The dashed lines show the predicted asymptotic behaviors (68). In the bottom figure we plot subtracted entanglement entropies to further check the large- $N$ convergence to Eq. (68), which is clearly demonstrated by the data.

Let us first consider a $2 \mathrm{D}$ system. We construct the ground state of a Fermi gases by filling all states with

$$
n_{1}+n_{2} \leq n_{e}, \quad n_{i}, n_{e}=1,2,3 \ldots
$$

The number $N$ of particles is a function of $n_{e}$, which asymptotically reads $N=n_{e}^{2} / 2$. Since the overlap matrix (60) is block diagonal, for any integer $k$ we have

$$
\begin{aligned}
& \operatorname{Tr}_{2 \mathrm{D}}\left[N\left(n_{e}\right)\right]^{k}=\sum_{n_{1}=1}^{n_{e}} \operatorname{Tr} \mathbb{A}_{1 \mathrm{D}}\left(n_{e}-n_{1}\right)^{k}, \\
& \mathbb{A}_{1 \mathrm{D}}(M)_{n m}=\int_{0}^{\infty} d z \phi_{n}(z) \phi_{m}(z),
\end{aligned}
$$

where $\mathbb{A}_{1 \mathrm{D}}(M)$ is the half-space $M \times M$ overlap matrix of the 1D system. This also implies analogous exact relations for all observables which can be constructed by traces of powers of the overlap matrix or from its eigen- values, such as the particle cumulants and the entanglement entropies, cf. Eqs. (24) and (28). Thus,

$$
\begin{aligned}
S_{\mathrm{HS}, 2 \mathrm{D}}^{(\alpha)}\left[N\left(n_{e}\right)\right] & =\sum_{n_{1}=1}^{n_{e}} S_{\mathrm{HS}, 1 \mathrm{D}}^{(\alpha)}\left(n_{e}-n_{1}\right), \\
V_{\mathrm{HS}, 2 \mathrm{D}}^{(m)}\left[N\left(n_{e}\right)\right] & =\sum_{n_{1}=1}^{n_{e}} V_{\mathrm{HS}, 1 \mathrm{D}}^{(m)}\left(n_{e}-n_{1}\right) .
\end{aligned}
$$

In order to derive their large- $N$ asymptotic behaviors, we replace the sums by integrals and use the relation $N=n_{e}^{2} / 2$, i.e.

$$
\begin{aligned}
& S_{\mathrm{HS}, 2 \mathrm{D}}^{(\alpha)}(N)=\int_{0}^{\sqrt{2 N}} d n S_{\mathrm{HS}, 1 \mathrm{D}}^{(\alpha)}(\sqrt{2 N}-n), \\
& V_{\mathrm{HS}, 2 \mathrm{D}}^{(m)}(N)=\int_{0}^{\sqrt{2 N}} V_{\mathrm{HS}, 1 \mathrm{D}}^{(m)}(\sqrt{2 N}-n) .
\end{aligned}
$$

Then we use the asymptotic formulas for the 1D quantities, cf. Eqs. (41), (42), (52), (53), obtaining

$$
\begin{aligned}
& S_{\mathrm{HS}, 2 \mathrm{D}}^{(\alpha)}(N) \approx a N^{1 / 2}\left[\ln N+a_{0}+o\left(N^{0}\right)\right], \\
& a=\frac{C_{\alpha}}{\sqrt{2}}=\frac{1+\alpha^{-1}}{12 \sqrt{2}}, \quad a_{0}=2 y_{\alpha}-2+7 \ln 2 .
\end{aligned}
$$

The approximations used to derive this asymptotic behavior from Eq. (64) should not affect the leading $O\left(N^{1 / 2} \ln N\right)$ and next-to-leading $O\left(N^{1 / 2}\right)$ term, so that the constants $a$ and $a_{0}$ should be considered as exact. This is confirmed by the analysis of the large- $N$ behavior of numerical data at fixed $N$. In Fig. 14we compare these asymptotic expansions with the data up to $N \approx 5000$ for the $\mathrm{vN}$ and $\alpha=2$ Rényi entropy, which clearly support them.

For the particle cumulants we obtain

$$
\begin{aligned}
& V_{\mathrm{HS}, 2 \mathrm{D}}(N)=v N^{1 / 2}\left[\ln N+v_{0}+o\left(N^{0}\right)\right], \\
& v=\frac{1}{2^{3 / 2} \pi^{2}}, \quad v_{0}=2 \gamma_{E}+7 \ln 2,
\end{aligned}
$$

and

$$
V_{\mathrm{HS}, 2 \mathrm{D}}^{(m)}(N) \approx \sqrt{2} \nu_{m} N^{1 / 2}, \quad m>2,
$$

where $\nu_{m}$ are the constants of the leading large- $N$ behavior in one dimension, cf. Eq. (54).

The above calculations can be straightforwardly extended to higher dimensions. In three dimensions we obtain

$$
S_{\mathrm{HS}, 3 \mathrm{D}}^{(\alpha)}(N) \approx \frac{C_{\alpha}}{\sqrt{6}} N^{2 / 3} \ln N .
$$

The above method can be also used to express the entanglement entropies and particle cumulants of the subsystems $B$ and $S$, defined at the end of Sec. IIB2, in terms of sum of $1 \mathrm{D}$ contributions for the interval $[-\infty, x]$ and $[-x, x]$ respectively. In particular, in the case of $2 \mathrm{D}$ 

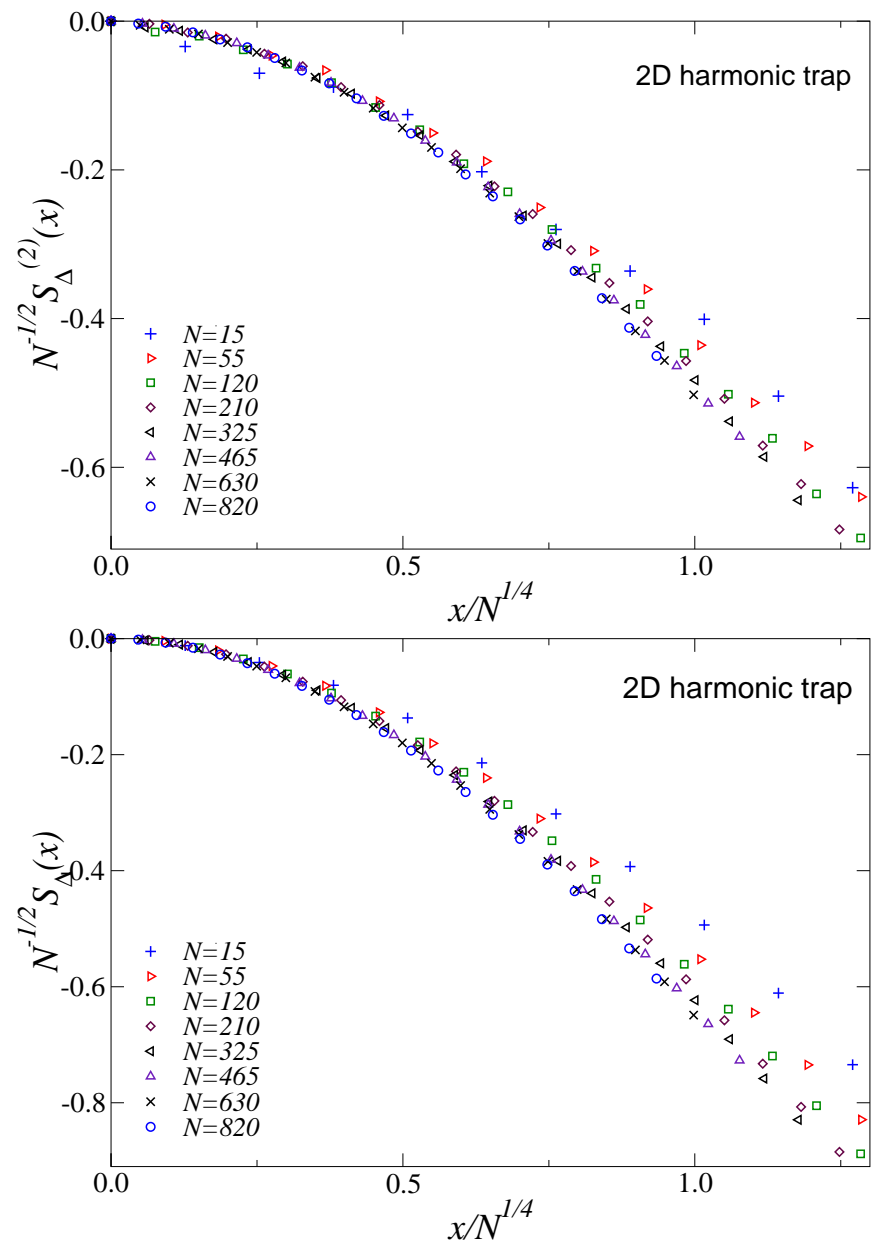

FIG. 15: (Color online) The space dependence of the $\alpha=$ 2 Rényi (top) and vN (bottom) entanglement entropies of $2 \mathrm{D}$ systems trapped by a harmonic potential. We plot $N^{-1 / 2} S_{\Delta}^{(\alpha)}(x)$ vs $x / N^{1 / 4}$ for the harmonic trap.

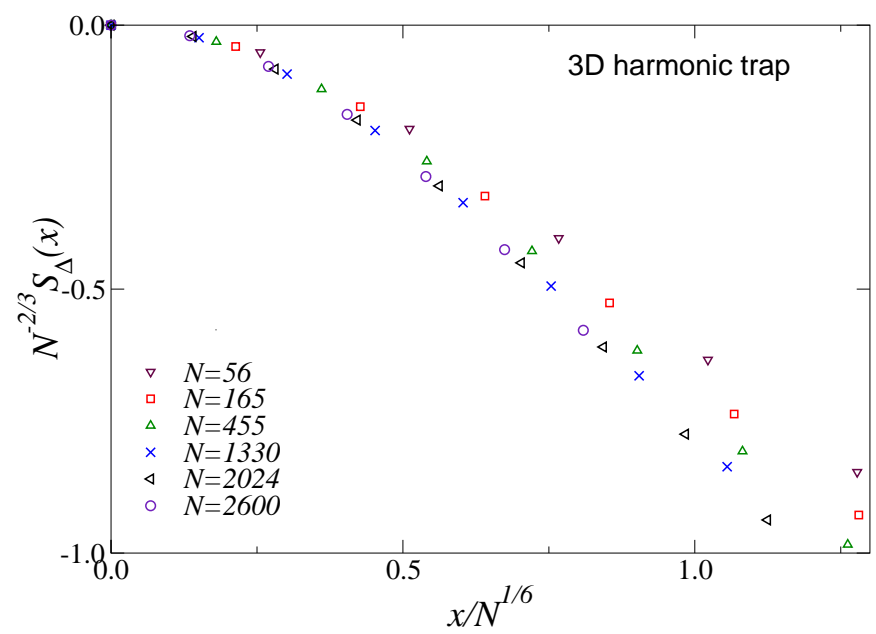

FIG. 16: (Color online) The space dependence of vN entanglement entropies of 3D systems trapped by a harmonic potential. We plot $N^{-2 / 3} S_{\Delta}^{(\alpha)}(x)$ vs $x / N^{1 / 6}$ for the harmonic trap. stripes $S$ contained within two parallel lines at distance $x$ from the center, the overlap matrix is

$$
\mathbb{A}_{n_{1}, \ldots, n_{d} ; m_{1}, \ldots, m_{d}}=\prod_{i=2}^{d} \delta_{n_{i} m_{i}} \int_{-x}^{x} d z \phi_{n_{1}}(z) \phi_{m_{1}}(z)
$$

which leads to equations analogous to Eqs. (62 65). Then, using the continuum approximation and the asymptotic large- $N$ behaviors of the 1D entanglement enetropies of the interval $[-x, x]$, cf. Eq. (48), we arrive at the asymptotic behavior

$$
S_{S, 2 \mathrm{D}}^{(\alpha)}(x) \approx \sqrt{2} C_{\alpha} N^{1 / 2}\left[\ln N+h_{S^{(\alpha)}}\left(x / N^{1 / 4}\right)\right] .
$$

The coefficient of the leading logarithmic term is just twice that of the half-space entanglement entropy (79), because the boundary of the stripe is double. Analogously, in three dimensions, we obtain

$$
S_{S, 3 \mathrm{D}}^{(\alpha)}(x) \approx \frac{\sqrt{2} C_{\alpha}}{\sqrt{3}} N^{2 / 3}\left[\ln N+h_{S^{(\alpha)}}\left(x / N^{1 / 6}\right)\right]
$$

Note that the large- $N$ scaling of the space variables depends on the spatial dimension $d$. For a generic $d$, it depends on the scaling variable $x / N^{1 /(2 d)}$.

The large- $N$ scaling of the space dependence of the entanglement entropies from the size of the extended space region can be also checked from the difference $S_{\Delta}(x)=$ $S_{B}(x)-S_{\mathrm{HS}}$ (we recall that the subsystem $B$ is separated from the rest by a hyperplane at a distance $x$ from the center of the trap). Fig. 15 shows numerical results for the $\alpha=1 \mathrm{vN}$ and the $\alpha=2$ Rényi entropyes, which support the large- $N$ scaling $S_{\Delta}^{(\alpha)}(x)=N^{1 / 2} f_{\Delta}^{(\alpha)}\left(x / N^{1 / 4}\right)$, i.e. the same large- $N$ scaling of the space dependence as in Eq. (73). Fig. 16 shows the $\mathrm{vN} S_{\Delta}(x)$ for $3 \mathrm{D}$ systems, which appear to scale as $S_{\Delta}(x)=N^{2 / 3} f_{\Delta}\left(x / N^{1 / 6}\right)$.

Analogous results are obtained for the particle variance. In particular, we find that the ratios of the coefficients of the leading terms in the asymptotic behaviors of the entanglement entropies and the particle variance satisfy the universal relation

$$
S_{A}^{(\alpha)} / V_{A} \approx \frac{\left(1+\alpha^{-1}\right) \pi^{2}}{6}+O(1 / \ln N)
$$

for any subsystem $A$ considered and in any dimension.

\section{CONCLUSIONS}

We investigate the quantum correlations arising in the ground state of free fermion gases trapped by an external space-dependent harmonic potential, $V \propto x^{2} / l^{2}$ where $l$ is the trap size, in one, two and three dimensions. We consider systems of $N$ particles, and focus on the large$N$ scaling behaviors of the quantum correlations, as inferred by the expectation values of product of local operators and bipartite entanglement entropies which quantify 
the nontrivial entanglement connections between different parts of extended quantum systems. In particular, we study the relations between the entanglement entropies and the cumulants of the particle distribution within the same extended subsystem, which can be obtained by integration of the particle-density correlations.

Our results for the large- $N$ behaviors of the particle density $\rho(x)$, the two-point particle correlation $C(x, y)$ and the connected density-density correlation $G_{n}(x, y)$, can be summarized by the following scaling equations:

$$
\rho(r) \approx N^{\theta} \xi^{-d} R_{\rho}\left(N^{(\theta-1) / d} r / \xi\right), \quad r \equiv|\mathbf{x}|
$$

and

$$
\begin{aligned}
& C\left(\mathbf{x}_{1}, \mathbf{x}_{2}\right) \approx N^{\theta} \xi^{-d} R_{C}\left(N^{\theta / d} \mathbf{x}_{1} / \xi, N^{\theta / d} \mathbf{x}_{\mathbf{2}} / \xi\right) \\
& G_{n}\left(\mathbf{x}_{1}, \mathbf{x}_{2}\right) \approx N^{2 \theta} \xi^{-2 d} R_{G}\left(N^{\theta / d} \mathbf{x}_{1} / \xi, N^{\theta / d} \mathbf{x}_{2} / \xi\right)
\end{aligned}
$$

for $\mathbf{x}_{1} \neq \mathbf{x}_{2}$, where $d$ is the spatial dimension of the system, $\xi \equiv l^{\theta}$ is the (oscillator) length scale induced by the trap, and $\theta=1 / 2$ is the trap exponent for the harmonic potential. The above large- $N$ behaviors are expected to also hold for higher power laws of the external potential, i.e. $V(r) \propto(r / l)^{p}$, by replacing the corresponding value of the trap exponent, i.e. $\theta \equiv p /(p+2)$. In the limit $p \rightarrow \infty$, corresponding to hard-wall trap, the scaling laws of homogeneous systems are recovered by setting $\theta=1$.

We compute and analyze the asymptotic large- $N$ behaviors of the particle cumulants and entanglement entropies of extended spatial regions. Our main results are:

(i) The half-space Rényi entanglement entropies behave as

$$
S_{\mathrm{HS}}^{(\alpha)}=\frac{1+\alpha^{-1}}{2} c_{l} N^{(d-1) / d}\left[\ln N+c_{0}+o(1)\right]
$$

which includes the vN entanglement entropy when $\alpha \rightarrow$ 1. In $1 \mathrm{D}$ systems, the constant of the logarithmic term is equal to that of the homogeneous system, i.e. $c_{l}=1 / 6$, which is related to the central charge $c=1$ of the corresponding conformal field theory [24, 35]. We also determine the subleading constant $c_{0}$, cf. Eqs. (41,42). We also obtain the constants $c_{l}$ and $c_{0}$ in higher dimensions, cf. Eqs. (68) and (71); in particular we find $c_{l}=1 /(6 \sqrt{2})$ and $c_{l}=1 /(6 \sqrt{6})$ for the leading logarithmic term in two and three dimensions respectively.

(ii) We compute the asymptotic large- $N$ behavior of the half-space particle cumulants. Only even cumulants are nonzero, because half-space odd cumulants vanish by symmetry. We obtain

$$
\begin{aligned}
& V_{\mathrm{HS}}=v_{l} N^{(d-1) / d}\left[\ln N+v_{0}+o(1)\right] \\
& V_{\mathrm{HS}}^{(2 k)} \approx w_{2 k} N^{(d-1) / d}, \quad k \geq 2
\end{aligned}
$$

In one dimension, see Eqs. (52) and (54), the constants of the leading terms $v_{l}$ and $w_{2 k}$ turn out to be equal to those of the homogeneous system with open boundary conditions (hard walls), which were already computed in Refs. [19] (see also Refs. [18, 48]). In particular, $v_{l}=$
$1 /\left(2 \pi^{2}\right)$ and $v_{0}$ is reported in Eq. (52). The constants $v_{l}$ and $v_{0}$ are also evaluated in higher dimensions, cf. Eqs. (69) and (70). Only the particle variance presents the leading logarithmic term, like homogeneous systems. We find that, in any dimension and for any subsystem $A$, the ratio of the coefficients of the leading terms in the entanglement entropies and particle variance satisfies the relation

$$
\frac{c_{l}}{v_{l}}=\frac{\pi^{2}}{3}
$$

(iii) We also consider spatial bipartitions with different geometries, in particular the entanglement entropy of a stripe $S$ around the center of the trap with the boundaries at a distance $x$ (in 1D $S=[-x, x])$, defined in Sec. II B 2. and studied its space dependence. We find the general behavior

$$
\begin{aligned}
S_{S}^{(\alpha)}(x) \approx & \frac{1+\alpha^{-1}}{2} 2 c_{l} N^{(d-1) / d}[\ln N+ \\
& \left.+f_{S^{(\alpha)}}\left(N^{(\theta-1) / d} x / \xi\right)\right]
\end{aligned}
$$

where $c_{l}$ is the same constant appearing in Eq. (779). The coefficient of the leading logarithmic term is just twice that of the half-space entanglement entropy (79), in any dimension, essentially because the boundary of the stripe is double. A detailed analysis of the 1D case is reported in Sec. III B 2, The particle variance shows an analogous behavior, i.e.

$$
V \approx 2 v_{l} N^{(d-1) / d}\left[\ln N++f_{V}\left(N^{(\theta-1) / d} x / \xi\right)\right],
$$

where $v_{l}$ is the same constant appearing in Eq. (80). Note that the large- $N$ scaling of the space dependence of the entanglement entropies and particle variance is analogous to that of the particle density, while it differs from that of the particle correlation $C(x, y)$ and the connected density correlation $G_{n}(x, y)$, cf. Eqs. (77) and (78).

The above results (i), (ii) and (iii) are consistent with the known asymptotic behaviors of homogeneous Fermi gases with open boundary conditions [13, 19, 26], obtainable by setting $\theta=p /(p+2) \rightarrow 1$.

The large- $N$ asymptotic behaviors are rapidly approached with increasing the number of particles. For example, in one dimension the behavior of $O\left(10^{2}\right)$, of even less, particles is already well approximated by the asymptotic behaviors.

Finally, a few comments are in order concerning the relations between particle cumulants and entanglement entropies of an extended subsystems $A$. For noninteracting fermions, one can write down a formal expansion of the entanglement entropies of bipartitions in terms of the even cumulants [14 17], such as

$$
\begin{aligned}
& S_{A}=\frac{\pi^{2}}{3} V_{A}+\frac{\pi^{4}}{45} V_{A}^{(4)}+\frac{2 \pi^{6}}{945} V_{A}^{(6)}+\ldots \\
& S_{A}^{(2)}=\frac{\pi^{2}}{4} V_{A}-\frac{\pi^{4}}{192} V_{A}^{(4)}+\frac{\pi^{6}}{23040} V_{A}^{(6)}+\ldots
\end{aligned}
$$


In homogeneous noninteracting fermion gases with $N$ particles in a finite volume of any dimension $d$, the above expansions gets effectively truncated in the large$N$ limit [19] because the high cumulants $V_{A}^{(m)}$ with $m>2$ are all suppressed relatively to the particle variance $V_{A}$. The leading $N^{(d-1) / d} \ln N$ asymptotic behavior of $S_{A}^{(\alpha)}$ in Eqs. (85) and (86) arises from $V_{A}$ only, because the leading order of each cumulant $V^{(k)}$ with $k>2$ vanishes for any subsystem $A$ (including disjoint ones) in any dimension. This implies the general asymptotic relation

$$
S_{A}^{(\alpha)} \approx \frac{\left(1+\alpha^{-1}\right) \pi^{2}}{6} V_{A} .
$$

Our results for Fermi gases trapped by a harmonic potential show an analogous scenario: the asymptotic relation (87) holds as well, in any dimension, significantly extending its validity.

We mention that the close relation between entanglement entropy and variance in noninteracting Fermi gas is also found in off-equilibrium phenomena after local quantum quenches [15, 18, 49], and in some dynamics regime of the off-equilibrium expansion of Fermi gases from a trap [50].

The situation is more involved for interacting systems. In systems with localized interactions arising from impurities [51], all the cumulants $V^{(2 k)}$ contribute to the asymptotic large- $N$ behavior of the entanglement entropies in the expansion $S_{A}^{(\alpha)}=\sum_{k=1}^{\infty} s_{k}^{(\alpha)} V_{A}^{(2 k)}$, although the expansion turns out to be rapidly converging [19]. The conservation of a global charge, and in particular the particle number, is crucial for the connections between bipartite entanglement and particle fluctuations. For interacting systems not conserving the particle number, the entanglement should be related to the more fundamental energy transport.

\section{Acknowledgments}

I thank Pasquale Calabrese and Mihail Mintchev for many useful discussions within common research projects.

\section{Appendix A: Asymptotic behavior of the 1D half-space entanglement entropies}

We consider a 1D lattice model of spinless fermions in the presence of an external space-dependent potential

$$
H_{f}==\sum_{\langle i j\rangle} c_{i}^{\dagger} h_{i j} c_{j}
$$

where $c_{i}$ is a spinless fermion operator, and

$$
h_{i j}=J\left(\delta_{i j}-\frac{1}{2} \delta_{i, j-1}-\frac{1}{2} \delta_{i, j+1}\right)+V\left(x_{i}\right) \delta_{i j} .
$$

We consider a power-law spatial dependence for the trapping potential,

$$
V(r)=\frac{1}{p} v^{p} r^{p},
$$

where $r$ is the distance from the center of the trap, $v$ is a positive constant and $p$ an even integer number. The trap size $l$ is defined as $l \equiv J^{1 / p} / v$. In the following we set $J=1$.

In one dimension this lattice free-fermion model can be exactly mapped into the hard-core (HC) limit of the Bose-Hubbard (BH) model, see, e.g., Ref. [52],

$$
\begin{aligned}
\mathcal{H}_{\mathrm{BH}}= & \frac{J}{2} \sum_{\langle i j\rangle}\left(b_{j}-b_{i}\right)^{\dagger}\left(b_{j}-b_{i}\right) \\
& +\frac{U}{2} \sum_{i} n_{i}\left(n_{i}-1\right)+\sum_{i} V\left(r_{i}\right) n_{i},
\end{aligned}
$$

where $\langle i j\rangle$ is the set of nearest-neighbor sites, $b_{i}$ are bosonic operators, $n_{i} \equiv b_{i}^{\dagger} b_{i}$ is the particle density operator, and $N=\left\langle\sum_{i} n_{i}\right\rangle$ is the particle number. The $\mathrm{HC}$ limit $U \rightarrow \infty$ of the $\mathrm{BH}$ model implies that the particle number $n_{i}$ per site is restricted to the values $n_{i}=0,1$. As a consequence of their exact mapping, the $\mathrm{HC} \mathrm{BH}$ and lattice free spinless fermions share the same particle density and density-density correlation, particle distribution of extended space regions, and also entanglement entropies of connected spatial bipartitions [12].

The large- $l$ limit keeping $N$ fixed differs from that performed at fixed chemical potential $\mu$, i.e., considering the BH Hamiltonian

$$
\mathcal{H}_{\mu}=\mathcal{H}_{\mathrm{BH}}+(\mu-1) \sum_{i} n_{i} .
$$

Indeed, the large trap-size limit, keeping $\mu$ fixed, implies an increase of the particle number so that

$$
N / l^{d}=\tilde{\rho}(\mu)
$$

asymptotically, where $d$ is the spatial dimension and $\tilde{\rho}(\mu)$ is a finite function of $\mu$. This thermodynamic limit is usually considered when quantum transitions are studied in confined particle systems. In the absence of the trap, the 1D HC BH model has three phases: the empty state for $\mu>1$ with $\left\langle n_{i}\right\rangle=0$, which may be seen as a particular $n=0$ Mott phase, a gapless superfluid phase for $|\mu|<1$, and a $n=1$ Mott phase for $\mu<-1$. See, e.g., Ref. [52].

We consider chains with even sites $L$ and open boundary conditions, and a trap of size $l$ centered between the middle sites of the chain. We divide the chain in two connected parts of length $l_{A}$ and $L-l_{A}$, and consider their Rényi entropies

$$
\mathcal{S}^{(\alpha)}\left(l_{A} ; L\right)=\mathcal{S}^{(\alpha)}\left(L-l_{A} ; L\right)=\frac{1}{1-\alpha} \ln \operatorname{Tr} \rho_{A}^{\alpha}
$$

where $\rho_{A}$ is the reduced density matrix of one of the two subsystems. Let us consider the half-space entanglement

$$
\mathcal{S}_{\mathrm{HS}}^{(\alpha)} \equiv S^{(\alpha)}(L / 2, L)
$$


Its large- $L$ behavior can be written as

$$
\begin{aligned}
\mathcal{S}_{\mathrm{HS}}^{(\alpha)}= & C_{\alpha}\left[\ln L+e_{\alpha}+O\left(L^{-1 / \alpha}\right)\right] \\
C_{\alpha}= & \frac{1+\alpha^{-1}}{12}, \\
e_{\alpha}= & \ln \sqrt{1-\mu^{2}}+\ln (4 / \pi)+y_{\alpha}, \\
y_{\alpha}= & \int_{0}^{\infty} \frac{d t}{t}\left[\frac{6}{1-\alpha^{-2}}\left(\frac{1}{\alpha \sinh t / \alpha}-\frac{1}{\sinh t}\right) \times\right. \\
& \left.\times \frac{1}{\sinh t}-e^{-2 t}\right]
\end{aligned}
$$

where $y_{\alpha}$ is given in Eq. (A12). This equation has been obtained using the results of Refs. [22, 35, 53, 54].

In a system confined by a trap of size $l$, we have [33]

$$
\begin{aligned}
& \mathcal{S}_{\mathrm{HS}}^{(\alpha)} \equiv \operatorname{Lim}_{L \rightarrow \infty} S^{(\alpha)}(L / 2 ; L)= \\
& =C_{\alpha}\left[\ln \xi_{e}+e_{\alpha}+O\left(\xi_{e}^{-1 / \alpha}\right)\right]
\end{aligned}
$$

where

$$
\xi_{e}=a_{e}(\mu) l
$$

is the entanglement length, which also enters the asymptotic formula of the energy difference of the two lowest states

$$
\Delta=\frac{\pi \sqrt{1-\mu^{2}}}{\xi_{e}} t(\phi), \quad t(\phi)=1 / 2-|\phi-1 / 2|,
$$

where $\phi$ is phase-like variable, $0 \leq \phi<1$, which parametrizes the modulations of the amplitude due to the periodic asymptotic occurrence of level crossings in the large- $l$ limit 36.

We now consider a $\mathrm{HC} \mathrm{BH}$ system of $N$ particles in a trap (centered in the middle of the chain), and we want to study the bipartite entanglement entropy, and in particular the half-lattice entanglement entropy, in the limit of large trap size (after taking the infinite chain limit $L \rightarrow \infty)$ as a function of $N$. More precisely, we consider

$$
\begin{aligned}
& S_{\mathrm{HS}}^{(\alpha)}(l, N)=\operatorname{Lim}_{L \rightarrow \infty} S^{(\alpha)}(L / 2, L ; l, N), \\
& S_{\mathrm{HS}}^{(\alpha)}(N) \equiv \operatorname{Lim}_{l \rightarrow \infty} S^{(\alpha)}(l, N),
\end{aligned}
$$

which is a finite function of $N$. Since $N$ is kept fixed while $l \rightarrow \infty$, this corresponds to the dilute regime $\mu \lesssim 1$. Let us define $\delta=1-\mu$, thus $\delta \rightarrow 0^{+}$corresponds to the dilution limit.

We want to derive asymptotic large- $N$ behavior of $S_{\mathrm{HS}}(N)$. For this purpose, we need: (i) the dependence of $a_{e}$ on $\mu$ when $\mu \rightarrow 1^{-}$; (ii) the relation between the number of particles and the chemical potential.

The dependence of $a_{e}$ on $\mu$ can be inferred from the behavior of the gap for $\delta \rightarrow 0$ (see Sec. IV B of Ref. 36]), by matching Eq. (A15) with its asymptotic behavior for $\delta \rightarrow 0$

$$
\Delta \approx c_{p} \delta^{(p-2) /(2 p)} \frac{t(\phi)}{l}
$$

where

$$
c_{p}(\delta)=\left\{\begin{array}{cll}
1 & \text { for } & p=2 \\
\frac{2 \sqrt{\pi} \Gamma(3 / 4)}{\Gamma(1 / 4)} & \text { for } & p=4 \\
\frac{\pi}{\sqrt{2}} & \text { for } & p \rightarrow \infty
\end{array}\right.
$$

In $1 \mathrm{D}$ particle systems, the thermodynamic limit at fixed $\mu$ corresponds to $N, l \rightarrow \infty$ keeping the ratio $N / l$ fixed. Indeed, we have

$$
N \equiv\left\langle\sum_{i} b_{i}^{\dagger} b_{i}\right\rangle=\tilde{\rho}(\mu) l+O(1)
$$

The function $\tilde{\rho}(\mu)$ can be computed in the HC limit. The particle density in the large- $l$ limit turns out to approach its local density approximation (LDA), with corrections that are suppressed by powers of the trap size and present a nontrivial TSS behaviour. Within the LDA, the particle density at the spatial coordinate $x$ equals the particle density of the homogeneous system at the effective chemical potential

$$
\mu_{\mathrm{eff}}(x) \equiv \mu+\frac{1}{p}\left(\frac{x}{l}\right)^{p} .
$$

The LDA of the particle density reads $\left\langle n_{x}\right\rangle_{\text {lda }} \equiv$ $\rho_{\text {lda }}(x / l)$, where

$\rho_{\text {lda }}(x / l)= \begin{cases}0 & \text { for } \mu_{\mathrm{eff}}(x)>1, \\ (1 / \pi) \arccos \mu_{\mathrm{eff}}(x) & \text { for }-1 \leq \mu_{\mathrm{eff}}(x) \leq 1, \\ 1 & \text { for } \mu_{\mathrm{eff}}(x)<-1\end{cases}$

Asymptotically, the total particle number is obtained by integrating the LDA of the particle density $\rho_{\text {lda }}$, obtaining

$$
\tilde{\rho}(\mu)=2 \int_{0}^{\infty} \rho_{\mathrm{lda}}(y) \mathrm{d} y .
$$

In the low-density regime, $\delta \equiv 1-\mu \rightarrow 0$,

$$
\tilde{\rho}(\mu)=r_{p} \delta^{(p+2) /(2 p)}[1+O(\delta)],
$$

with

$$
r_{p}=\left\{\begin{array}{cll}
1 & \text { for } & p=2 \\
\frac{2 \Gamma(1 / 4)}{3 \sqrt{\pi} \Gamma(3 / 4)} & \text { for } & p=4 \\
\frac{2 \sqrt{2}}{\pi} & \text { for } & p \rightarrow \infty
\end{array}\right.
$$

Using these results we obtain the entanglement entropy (A13) in terms of $N$ :

$$
S_{\mathrm{HS}}^{(\alpha)}(N)=C_{\alpha}\left[\ln N+\ln \frac{4(p+2)}{p}+y_{\alpha}+o\left(N^{0}\right)\right] .
$$

The above formula can be compared with large- $L$ and then large- $l$ extrapolations of numerical results by exact diagonalization of chains of size $L$ and traps of size $l$ 
centered between the middle sites. In particular, the $N$ dependent limit (A26) is approached with $O\left(l^{-2 \theta}\right)$ corrections, where $\theta=p /(p+2)$ is the trap exponent, conferming general theoretical arguments on the large- $l$ corrections [32].

The large trap-size limit keeping the particle number $N$ fixed corresponds to the dilute limit of continuum models in the presence of the trapping potential. As argued in Ref. [32], it also represents the asymptotic large- $N$ behavior for finite on-site couplings $U>0$ within the $\mathrm{BH}$ model, or finite-strength models of bosonic gases with short-range interactions.
[1] E.A. Cornell, C.E. Wieman, Rev. Mod. Phys. 74, 875 (2002).

[2] N. Ketterle, Rev. Mod. Phys. 74, 1131 (2002).

[3] I. Bloch, J. Dalibard, and W. Zwerger, Rev. Mod. Phys. 80,885 (2008).

[4] T. Kinoshita, T. Wenger, and D.S. Weiss, Nature 440, 900 (2006).

[5] T. Kinoshita, T. Wenger, and D.S. Weiss, Science 305, 1125 (2004); Phys. Rev. Lett. 95, 190406 (2005).

[6] T. Stöferle, H. Moritz, C. Schori, M. Köhl, and T. Esslinger, Phys. Rev. Lett. 92, 130403 (2004).

[7] B. Paredes, A. Widera, V. Murg, O. Mandel, S. Fölling, I. Cirac, G. Shlyapnikov, R.W. Hänsch, and I. Bloch, Nature 429, 277 (2004).

[8] B. Laburthe Tolra, K.M. O'Hara, J.H. Huckans, S.L. Rolston, and J.V. Porto, Phys. Rev. Lett. 92, 190401 (2004).

[9] S. Hofferberth, I. Lesanovsky, B. Fischer, T. Schumm, and J. Schmiedmayer, Nature 449, 324 (2007).

[10] L. Amico, R. Fazio, A. Osterloh, and V. Vedral, Rev. Mod. Phys. 80, 517 (2008).

[11] J. Eisert, M. Cramer, and M. B. Plenio, Rev. Mod. Phys. 82,277 (2010).

[12] Entanglement entropy in extended systems, P. Calabrese, J. Cardy, and B. Doyon Eds, J. Phys. A 42, 500301 (2009).

[13] P. Calabrese, M. Mintchev, and E. Vicari, EPL 97, 20009 (2012).

[14] I. Klich, G. Refael, and A. Silva, Phys. Rev. A 74, 032306 (2006).

[15] I. Klich and L. Levitov, Phys. Rev. Lett. 102, 100502 (2009).

[16] H.F. Song, S. Rachel, and K. Le Hur, Phys. Rev. B 82, 012405 (2010).

[17] H.F. Song, C. Flindt, S. Rachel, I. Klich, and K. Le Hur, Phys. Rev. B 83, 161408 (2011).

[18] H.F. Song, S. Rachel, C. Flindt, I. Klich, N. Laflorencie, and K. Le Hur, Phys. Rev. B 85, 035409 (2012).

[19] P. Calabrese, M. Mintchev, and E. Vicari, EPL in press, arXiv:1111.4836.

[20] M. Campostrini and E. Vicari, Phys. Rev. A 81, 023606 (2010); Phys. Rev. Lett. 102, 240601 (2009); 103, 269901 (2009) (E).

[21] E. Lukacs, Characteristic functions (C. Griffin, London 1970).

[22] B-Q Jin and V.E. Korepin, J. Stat. Phys. 116, 79 (2004).

[23] I. Peschel and V. Eisler, J. Phys. A 42, 504003 (2009).

[24] P. Calabrese, M. Mintchev, and E. Vicari, Phys. Rev. Lett. 107, 020601 (2011).

[25] I. Klich, J. Phys. A 39, L85 (2006).

[26] P. Calabrese, M. Mintchev, and E. Vicari, J. Stat. Mech.
P09028 (2011)

[27] E.H. Lieb and W. Liniger, Phys. Rev. 130, 1605 (1963).

[28] M. Girardeau, J. Math. Phys. (N.Y.) 1, 516 (1960); M.D. Girardeau, Phys. Rev. 139, B500 (1965).

[29] D.S. Petrov, G.V. Shlyapnikov, and J.T.M. Walraven, Phys. Rev. Lett. 85, 3745 (2000).

[30] F. Kalish and D. Braak, J. Phys. A 35, 9957 (2002).

[31] T.M. Garoni, P.J. Forrester, and N.E. Frankel, J. Math. Phys. 46, 103301 (2005).

[32] M. Campostrini and E. Vicari, Phys. Rev. A 82, 063636 (2010).

[33] M. Campostrini and E. Vicari, J. Stat. Mech. P08020 (2010); ibid E04001 (2001) (E).

[34] P.J. Forrester, Nucl. Phys. B 402, 709 (1993).

[35] P. Calabrese and J. Cardy, J. Stat. Mech. P06002 (2004).

[36] M. Campostrini and E. Vicari, Phys. Rev. A 81, 063614 (2010).

[37] V.V. Franca and K. Capelle, Phys. Rev. Lett. 100, 070403 (2008).

[38] J.S. Silva-Valencia and A.M.C. Souza, Phys. Rev. A 85, 033612 (2012).

[39] H. Widom, Toeplitz centennial (Tel Aviv 1981), Operator Theory: Adv. Appl. 4 (Birkhäuser, Basel, Boston, MA, 1982).

[40] M. Wolf, Phys. Rev. Lett. 96, 010404 (2006).

[41] D. Gioev and I. Klich, Phys. Rev. Lett. 96, 100503 (2006).

[42] T. Barthel, M.-C. Chung, and U. Schollwöck, Phys. Rev. A 74, 022329 (2006).

[43] W. Li, L. Ding, R. Yu, T. Roschilde, and S. Haas, Phys. Rev. B 74, 073103 (2006).

[44] S. Farkas and Z. Zimboras, J. Math. Phys. 48, 102110 (2007).

[45] R. Helling, H. Leschke, and W. Spitzer, Int. Math. Res. Not. 2011, 1451 (2011).

[46] L. Ding, N. Bray-Ali, R. Yu, and S. Haas, Phys. Rev. Lett. 100, 215701 (2008).

[47] B. Swingle, Phys. Rev. Lett. 105, 050502 (2010).

[48] V. Eisler, Ö. Legeza, and Z. Rácz, J. Stat. Mech. P11013 (2006).

[49] B. Hsu, E. Grosfeld, and E. Fradkin, Phys. Rev. B 80, 235412 (2009).

[50] E. Vicari, in preparation.

[51] P. Calabrese, M. Mintchev, and E. Vicari, J. Phys. A 45, 105206 (2012).

[52] S. Sachdev, Quantum Phase Transitions (Cambridge Univ. Press, 1999).

[53] F. Iglói and R. Juhász, EPL 8157003 (2008)

[54] P. Calabrese and J. Cardy, J. Stat. Mech. P04023 (2010). 